\title{
Article \\ Spectrum of Genetic Diseases in Tunisia: Current Situation and Main Milestones Achieved
}

\author{
Nessrine Mezzi ${ }^{1,2}$, Olfa Messaoud ${ }^{1}$ (D), Rahma Mkaouar ${ }^{1}$, Nadia Zitouna ${ }^{1}$, Safa Romdhane ${ }^{1}$, \\ Ghaith Abdessalem 1 ${ }^{1}$ Cherine Charfeddine 1,3 ${ }^{\mathbb{D}}$, Faouzi Maazoul ${ }^{4}$, Ines Ouerteni ${ }^{4}$, Yosr Hamdi 1,5, \\ Anissa Zaouak ${ }^{6}$, Ridha Mrad ${ }^{4}$, Sonia Abdelhak ${ }^{1}$ (D) and Lilia Romdhane ${ }^{1,2, *(D)}$
}

1 Laboratory of Biomedical Genomics and Oncogenetics, Institut Pasteur de Tunis, Tunis 1002, Tunisia; nesrine.mezzi@pasteur.utm.tn (N.M.); olfa.messaoud@pasteur.tn (O.M.); rahma.mkaouar@fst.utm.tn (R.M.); zitounanadia@gmail.com (N.Z.); safa.romdhane@pasteur.utm.tn (S.R.);

ghaith.abdessalem@pasteur.utm.tn (G.A.); cherine.charfeddine@gmail.com (C.C.); yosr.hamdi@pasteur.utm.tn (Y.H.); sonia.abdelhak@pasteur.utm.tn (S.A.)

2 Department of Biology, Faculty of Sciences of Bizerte, Université Tunis Carthage, Jarzouna 7021, Tunisia

3 High Institute of Biotechnology of Sidi Thabet, Biotechpole of Sidi Thabet, University of Manouba, Ariana 2080, Tunisia

4 Department of Congenital and Hereditary Diseases, Charles Nicolle Hospital, Tunis 1002, Tunisia; f.maazoul@yahoo.fr (F.M.); ines_ouertani@yahoo.fr (I.O.); ridhamr@rns.tn (R.M.)

5 Laboratory of Human and Experimental Pathology, Institut Pasteur de Tunis, Tunis 1002, Tunisia

check for updates

Citation: Mezzi, N.; Messaoud, O.; Mkaouar, R.; Zitouna, N.; Romdhane, S.; Abdessalem, G.; Charfeddine, C.; Maazoul, F.; Ouerteni, I.; Hamdi, Y.; et al. Spectrum of Genetic Diseases in Tunisia: Current Situation and Main Milestones Achieved. Genes 2021, 12, 1820. https://doi.org/10.3390/ genes12111820

Academic

Editors: Domenico Coviello, Pablo Lapunzina, Zilfalil Alwi and Ghada El Kamah

Received: 27 September 2021 Accepted: 3 November 2021 Published: 19 November 2021

Publisher's Note: MDPI stays neutral with regard to jurisdictional claims in published maps and institutional affiliations.

Copyright: (c) 2021 by the authors. Licensee MDPI, Basel, Switzerland. This article is an open access article distributed under the terms and conditions of the Creative Commons Attribution (CC BY) license (https:// creativecommons.org/licenses/by/ $4.0 /)$.
6 Department of Dermatology, Research Unit Genodermatosis and Cancer LR12SP03, Habib Thameur Hospital, Faculty of Medicine of Tunis, University of Tunis El Manar, Tunis 1002, Tunisia; anissa_zaouak@yahoo.fr

* Correspondence: lilia.romdhane@pasteur.utm.tn; Tel.: +216-97464780

Abstract: Genetic diseases in Tunisia are a real public health problem given their chronicity and the lack of knowledge concerning their prevalence and etiology, and the high rates of consanguinity. Hence, we performed systematic reviews of the literature in order to provide a more recent spectrum of these disorders and to expose the challenges that still exist to tackle these kinds of diseases. A manual textual data mining was conducted using MeSH and PubMed databases. Collected data were classified according to the CIM-10 classification and the transmission mode. The spectrum of these diseases is estimated to be 589 entities. This suggests remarkable progress through the development of biomedical health research activities and building capacities. Sixty percent of the reported disorders are autosomal recessive, which could be explained by the high prevalence of endogamous mating. Congenital malformations (29.54\%) are the major disease group, followed by metabolic diseases $(22 \%)$. Sixty percent of the genetic diseases have a known molecular etiology. We also reported additional cases of comorbidity that seem to be a common phenomenon in our population. We also noticed that epidemiological data are scarce. Newborn and carrier screening was only limited to pilot projects for a few genetic diseases. Collected data are being integrated into a database under construction that will be a valuable decision-making tool. This study provides the current situation of genetic diseases in Tunisia and highlights their particularities. Early detection of the disease is important to initiate critical intervention and to reduce morbidity and mortality.

Keywords: genetic diseases; Tunisian population; public health; consanguinity; database

\section{Introduction}

Rare diseases (RDs) are defined differently from one jurisdiction or organization to another [1]. In the USA, a rare disease (RD) is a disease affecting fewer than 200,000 people in the population (https://rarediseases.info.nih.gov, accessed on 24 September 2021). In Europe, a prevalence of less than 1/2000 people was specified to define a RD [2]. In Japan, any disorder affecting 1 in 2500 people is a RD [3]. In an attempt to define a consensus about an international definition of RD, Ritcher and his colleagues proposed a global average of 
40 cases /100,000 people with an average of $1 / 2500$ people [3]. In the Arab world, including Tunisia, there is no standard definition of RD. RDs imply a great global burden and a significant challenge for health systems resulting from unmatched patients' needs and the absence of equal access to diagnosis and treatment [4]. Nearly 6000 RDs have been identified around the world (https:/ / www.rarediseaseday.org/, accessed on 24 September 2021). Only $5 \%$ of them have an FDA-approved treatment (https: / / globalgenes.org/rare-diseasesfacts-statistics /, accessed on 24 September 2021).

Approximately $72 \%$ of RDs are attributed to genetic factors (https: / / www.rarediseaseday. org/, accessed on 24 September 2021). Genetic diseases are generally early-onset and lifethreatening conditions [5]. About 30\% of affected individuals die before five years [5]. Genetic diseases particularly concern the Arab world, where at least 1890 disorders have been identified (https:/ / www.cags.org.ae/en, accessed on 24 September 2021). Congenital and genetic diseases are a major cause of infant mortality and handicap among Arab countries [6]. The spread of genetic diseases in the Arab world, especially rare conditions, was largely explained by socio-cultural factors $[7,8]$. Indeed, the highest rates of consanguinity with an extreme prevalence of first-cousin unions were described among Arab families, also known by their large size [9]. In addition, high paternal and maternal age have also been documented among Arabs [6].

Tunisia, a middle-income country, is situated in a strategic position at the crossroad of Europe, the Middle East and Sub-Saharan Africa. The current population is estimated to be $11,860,516$ in November 2020, with a population growth rate assessed to $1.11 \%$ (https: //tradingeconomics.com/tunisia; https://www.worldometers.info/world-population, accessed on 24 September 2021). About $98 \%$ of Tunisians are Muslims living with other minorities, such as Christians and Jews [10]. The country has been inhabited since the Paleolithic period [11]. Berbers, also called Amazigh, are the indigenous people of Tunisia $[12,13]$. This has been well confirmed by the investigation of the HLA class I and class II gene profiles among Tunisians, which shows the Berber origin of the present-day Tunisian population [14]. Throughout its history, the Tunisian population has witnessed successive invasions and several migratory flows during prehistoric and historic periods [13], thus shaping its genetic landscape [10].

Tunisia is characterized by the improvement of health system indicators [15]. In the MENA region, Tunisia has the highest life expectancy with remarkable improvements in maternal and infant mortality [16]. This could be explained by the implementation of social programs aiming to reduce the burden of communicable diseases (CD) [15] Consequently, epidemiological data show that non-communicable diseases (NCD), such as genetic disorders, exceed CD [17]. To a certain extent, this can be explained by changes in national economic activities, family structure, and lifestyle that could foster the rising burden of NCD [18]. Similarly to other Arab countries, familial and geographic endogamy is a deeply conserved rule in Tunisia with remarkable historical and social roots with rates ranging up to $38.0 \%$ despite the remarkable socio-demographic changes [7,19-21]. This socio-cultural feature has been found to be strongly associated with the expression of rare genetic diseases $[19,22]$. In a previous study, we reported on the preliminary spectrum of genetic diseases in Tunisia. Such conditions are considered as real problems of public health in Tunisia as their spectrum encompasses 346 genetic disorders [23]. A large proportion is represented by autosomal recessive diseases $(62.9 \%)$, followed by autosomal dominant diseases highlighting the impact of inbreeding in their emergence [23]. In the present work, we performed a systematic review of the literature relative to genetic diseases in Tunisia in order to (i) "provide a more recent spectrum" of these disorders in the Tunisian population, (ii) identify the type of potential health interventions that could be proposed for these conditions in Tunisia, and (iii) to shed light on challenges that still exist. 


\section{Materials and Methods}

\subsection{Systematic Review of Literature Related to Genetic Diseases Spectrum in Tunisia}

A comprehensive review of published data was conducted mainly using the MeSH and PubMed databases. The list of genetic diseases was obtained after querying the MeSH database using the keywords "genetic diseases". The query results were further combined to additional keywords "tunis" using the PubMed search tool. We focused only on papers published from 2010 to August 2021. The final query on PubMed was the following: ((“Genetic Diseases, Inborn”[Mesh]) AND (Tunis*)) AND ((“2010”[Date-Publication]: “2021"[Date-Publication])).

The retained papers $(n=970)$ were included in the meta-analysis and were curated manually. They were completely screened for disease name, OMIM ID, inheritance mode, associated gene(s), germinal mutation(s) (according to HGVS nomenclature), and epidemiological parameters (prevalence, incidence, and patient counts). We also searched for the same information from the grey literature (including technical or research reports, doctoral thesis, conference proceedings, etc.). From the selected documents reporting on genetic diseases in the Tunisian population, a recursive search for additional documents was performed using the authors' names of referring physicians and researchers in Tunisia.

We used the tenth version of the World Health Organization (WHO) International Classification of Disease (WHO ICD-10) in order to provide a clustering of the genetic diseases in different pathological groups. Our previous data on the genetic diseases' spectrum published in 2011 have been compared to the updated data using the Fisher test, considering $5 \%$ as a statistical significance threshold. The data have been stored in a local database for subsequent analyses (Figure 1).

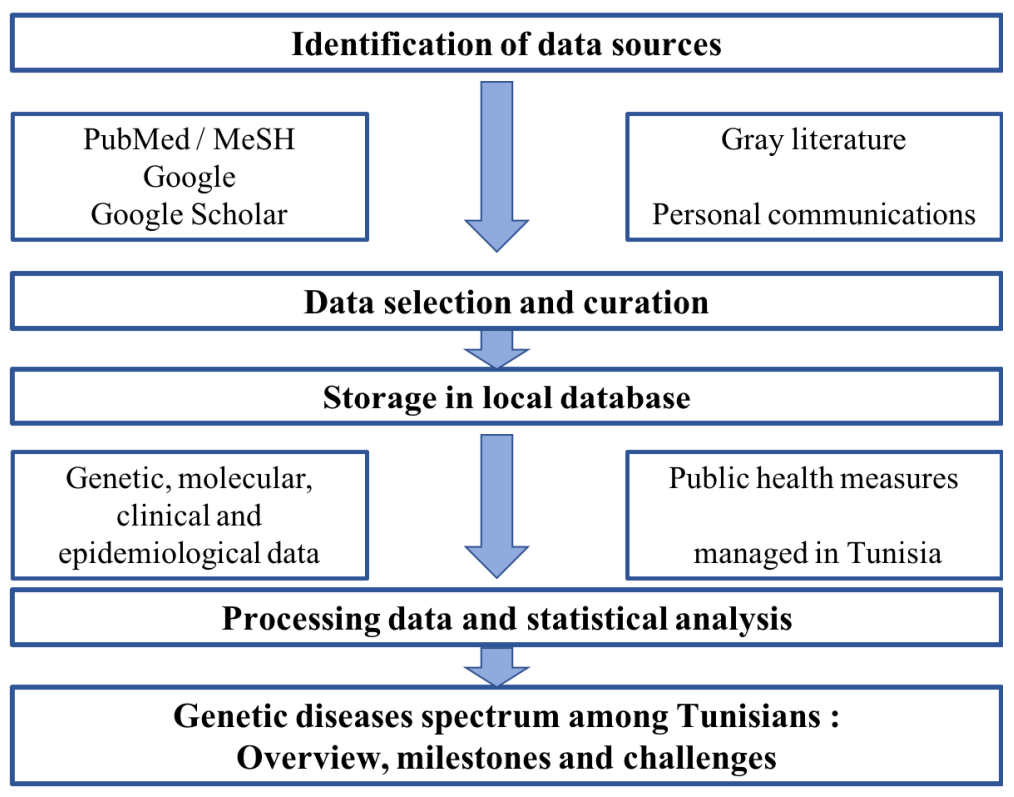

Figure 1. Screening process workflow.

2.2. Systematic Review of Literature Focusing on Newborn and/or Carrier Screening for Genetic Diseases among Tunisians

To get knowledge about the current genetic disease screening situation in Tunisia, a selective literature search was carried out. Multiple keywords have been used to develop an advanced PubMed query string as below:

("Genetic Diseases, Inborn"[Mesh]) AND (("Mass Screening"[Mesh] OR “Diagnosis"[Mesh] OR "Maternal Serum Screening Tests"[Mesh] OR “High-Throughput Screening Assays"[Mesh] OR "Neonatal Screening"[Mesh] OR "Multiphasic Screening"[Mesh] OR "Genetic Carrier Screening" [Mesh] OR "Diagnostic Screening Programs"[Mesh] OR “DirectTo-Consumer Screening and Testing"[Mesh] OR "Prenatal Diagnosis"[Mesh] OR "Nonin- 
vasive Prenatal Testing"[Mesh] OR “Preimplantation Diagnosis"[Mesh] OR “Mandatory Testing"[Mesh]) AND (Tunis*))

Only studies dealing with genetic disease screening among Tunisians were kept. In addition, we asked referring experts (clinicians, researchers) with whom we collaborate in order to provide more information concerning screening for these disorders. Moreover, grey literature was an important resource in this part of the systematic review.

Furthermore, we have defined a list of genetic diseases frequently described among Tunisians based on epidemiological data, essentially patients' counts. The experience feedback of our collaborators was of great value in this step of analysis. We have checked the eligibility of the identified commonly-reported genetic diseases for systematic newborn and / or carrier screening in our country by responding to key questions relative to newborn screening and using the seven ACOG carrier-screening criteria mentioned in their committee opinion in 2017. These criteria are the following (a) well-defined phenotype, (b) detrimental effect on the quality of life, (c) cognitive or physical impairment, (d) surgical or medical intervention is required, (e) an early life onset, (f) availability of prenatal diagnosis, and $(\mathrm{g})$ carrier frequency of 1 in 100 or greater. Evaluation of these criteria was conducted by a genetic counselor.

\section{Results}

In this study, we present the actual situation of genetic diseases in Tunisia. Compared to what has been published in 2011, 243 additional genetic conditions have been identified, with an average of approximately 23 genetic diseases reported every year. Thus, the total number of genetic diseases in Tunisia amounts to 589 clinical and/or genetic entities, in which $41.3 \%$ come from the present data mining.

\subsection{Classification of Genetic Diseases According to WHO-ICD 10}

We opted for the international medical classification (WHO ICD-10) of genetic diseases described in the Tunisian population. Therefore, such classification revealed that congenital malformations, deformations, and chromosomal abnormalities are the most commonly reported $(29.54 \%)$. Endocrine, nutritional, and metabolic diseases are the next most frequent group of diseases (22\%), followed by diseases of the nervous system (15.45\%) (Figure 2). We report a slight increase in the following disease groups: diseases of the ear and mastoid process and diseases of the eye and adnexa. We also noticed that diseases of the circulatory system are highly reported. A comparison between genetic diseases spectrum data of 2011 and the updated data of our study highlighted a significant difference in the distribution of disease classes $\left(\right.$ Fisher test $p$-value $=9.9 \times 10^{-4}$ ).

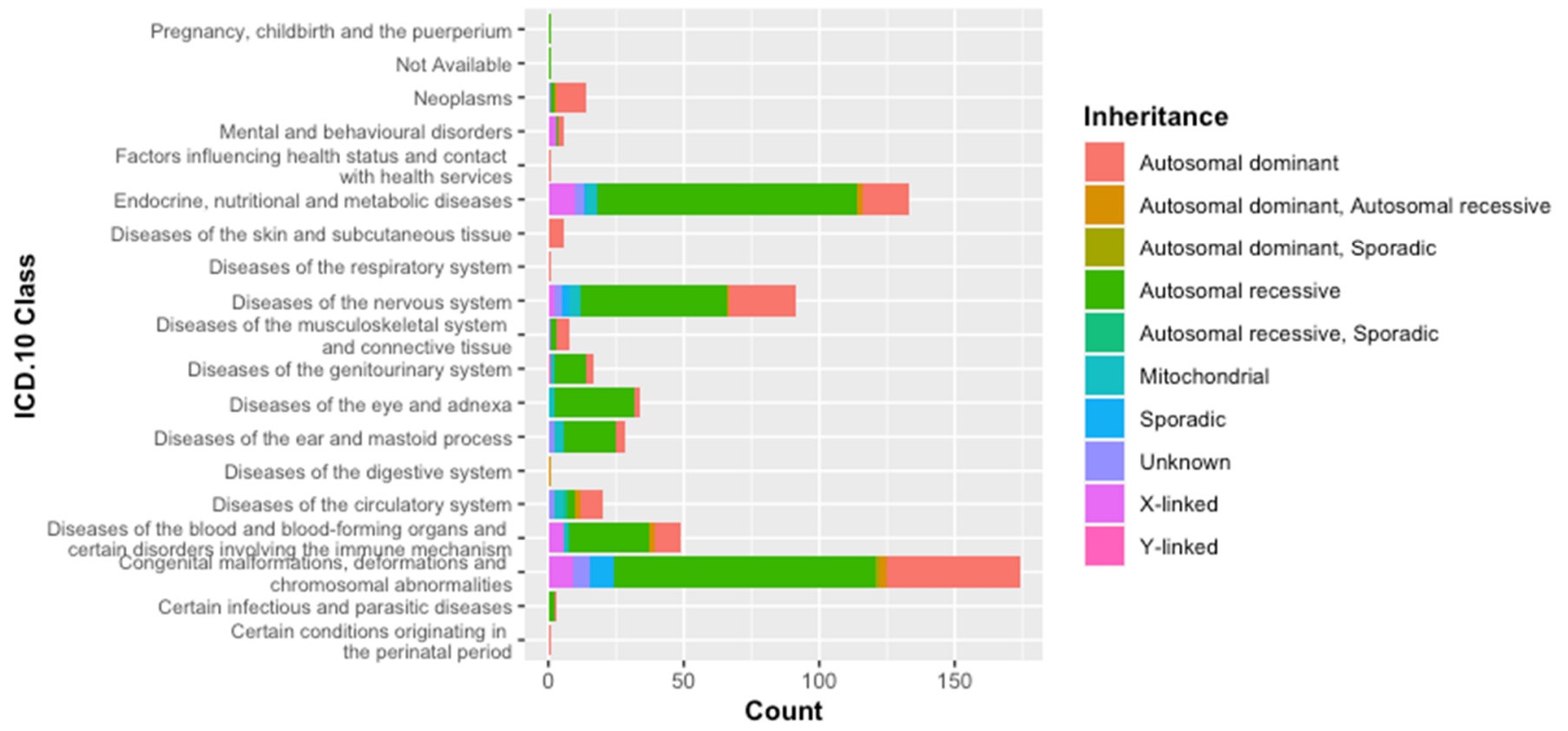

Figure 2. WHO ICD-10 classification of genetic diseases. 


\subsection{Classification According to the Inheritance Mode}

Among genetic diseases derived from this analysis (243 genetic diseases), autosomal recessive diseases (AR) are the most frequent $(54.7 \%)$, followed by autosomal dominant $(\mathrm{AD})$ disorders $(27.16 \%)$. X-linked $(\mathrm{XL})$ conditions were encountered $5 \%$ of the time. Diseases transmitted with both $\mathrm{AR}$ and $\mathrm{AD}(\mathrm{AR}, \mathrm{AD})$ are more prevalent than in data reported before 2011. Remarkably, diseases inherited via mitochondrial mode are becoming more reported $(8.2 \%)$. In order to check for an eventual change in the inheritance mode distribution between the two datasets, we have compared our current data to that of the previous spectrum. We found a significant difference (Fisher's test; $p$-value $=5 \times 10^{-4}$ ). Despite this difference, the whole spectrum of genetic diseases described among Tunisians (589 genetic diseases) has kept the same pattern illustrated by the high frequency of AR diseases (60\%) followed by the AD conditions (24.3\%) (Figure 2).

\subsection{Molecular Etiology of the Genetic Diseases in the Tunisian Population}

Sixty-one percent (61\%) of the 589 genetic diseases have a known molecular etiology (358 genetic diseases), meaning that the responsible gene with at least one mutation has been identified among Tunisian patients. About $69 \%$ of these diseases are autosomal recessive, $16.2 \%$ are autosomal dominant, $7 \%$ are caused by a mutation in mitochondrial genes, $6 \%$ are $\mathrm{X}$-linked, and only $0.3 \%$ are $\mathrm{Y}$-linked or sporadic. Autosomal dominant or autosomal recessive diseases are encountered at $1.2 \%$ (Figure 3 ).

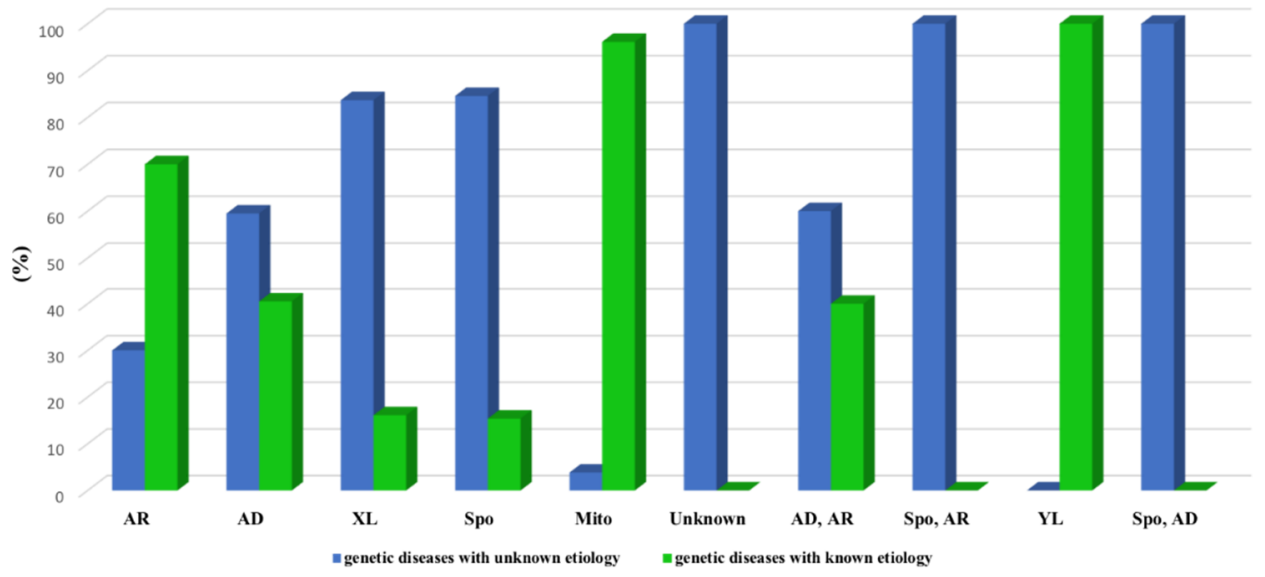

Figure 3. Classification according to the inheritance mode of the whole spectrum of genetic disorders (AR: Autosomal Recessive; AD: Autosomal Dominant; XL: X-linked; Spo: Sporadic; Mito: Mitochondrial; YL: Y-linked).

Among these diseases, 21 conditions were of unknown molecular etiology before the last decade (reported in the study of Romdhane and Abdelhak, 2011) (Table 1). Eleven diseases mainly belong to the endocrine, nutritional, and metabolic disorders class. Three are diseases of the nervous system, and one is a bleeding disorder. The eight remaining correspond to congenital malformations, deformations, and chromosomal abnormalities (Table 1). Next-generation sequencing has been used to elucidate the molecular etiology in four of them (Table 1). Referring to the original study conducted by Romdhane and Abdelhak in 2011, the Tunisian mutational spectrum included 420 mutations (98\% nuclear, $2 \%$ mitochondrial) (Romdhane et al. 2011) (23). Here, the global spectrum comprises more than 896 mutations. These mutations affect 313 genes: $95.5 \%$ nuclear (299 genes) and $4.5 \%$ mitochondrial (14 genes). The actual Tunisian mutational spectrum encompasses $429(48 \%)$ founder mutations responsible for 197 genetic diseases. Allelic heterogeneity was noticed for 154 genes (Table S1 Supplementary Data). HBB, G6PD, and CFTR genes were the most mutated genes among Tunisians with at least 25 alterations. 
Table 1. Diseases with an identified molecular etiology determined in the last decade.

\begin{tabular}{|c|c|c|c|c|c|c|c|}
\hline Disease Name & OMIM & Inheritance & Gene (OMIM) & Genetic Variant(s) & ICD-10 Classification & $\begin{array}{l}\text { Approach Used } \\
\text { for Molecular } \\
\text { Diagnosis }\end{array}$ & References \\
\hline \multirow{2}{*}{$\begin{array}{c}\text { Adrenal Hyperplasia, } \\
\text { Congenital, due to } \\
11-\beta \text {-Hydroxylase Deficiency }\end{array}$} & \multirow{2}{*}{202010} & \multirow{2}{*}{$\mathrm{AR}$} & \multirow{2}{*}{$\begin{array}{l}\text { CYP11B1 } \\
(610613)\end{array}$} & $\begin{array}{c}\text { c.1066C > T } \\
\text { (p.Gln356Ter) }\end{array}$ & \multirow{2}{*}{$\begin{array}{l}\text { Endocrine, nutritional, and } \\
\text { metabolic diseases }\end{array}$} & \multirow{2}{*}{$\begin{array}{l}\text { Sanger } \\
\text { sequencing }\end{array}$} & \multirow{2}{*}{ [24] } \\
\hline & & & & $\begin{array}{c}\text { c.1136G > T } \\
\text { (p.Gly379Val) }\end{array}$ & & & \\
\hline Adrenoleukodystrophy & 300100 & $\mathrm{XL}$ & $\begin{array}{l}\text { ABCD1 } \\
(300371)\end{array}$ & c.284C > A (p.Ala95Asp) & $\begin{array}{l}\text { Endocrine, nutritional, and } \\
\text { metabolic diseases }\end{array}$ & $\begin{array}{c}\text { Sanger } \\
\text { sequencing }\end{array}$ & [25] \\
\hline Alstrom syndrome & 203800 & AR & $\begin{array}{l}\text { ALMS } 1 \\
(606844)\end{array}$ & c. $10388-2 A>G$ & $\begin{array}{c}\text { Congenital malformations, } \\
\text { deformations, and chromosomal } \\
\text { abnormalities }\end{array}$ & $\begin{array}{l}\text { Targeted Gene } \\
\text { sequencing }\end{array}$ & [26] \\
\hline Bloom syndrome & 210900 & $\mathrm{AR}$ & $B L M(604610)$ & $\begin{array}{l}\text { c.1980-1982delAA } \\
\text { (p.Lys662fsX5) }\end{array}$ & $\begin{array}{c}\text { Congenital malformations, } \\
\text { deformations, and chromosomal } \\
\text { abnormalities }\end{array}$ & $\begin{array}{c}\text { Sanger } \\
\text { sequencing }\end{array}$ & $\begin{array}{l}\text { Ezzine et al., } \\
\text { Unpublished } \\
\text { [27] }\end{array}$ \\
\hline \multirow{8}{*}{ FVII deficiency } & \multirow{8}{*}{227500} & \multirow{8}{*}{$\mathrm{AR}$} & \multirow{8}{*}{$F 7(613878)$} & $\begin{array}{c}\text { c.90-91insA } \\
\text { (p.12LeufsX11) }\end{array}$ & \multirow{8}{*}{$\begin{array}{l}\text { Diseases of the blood and } \\
\text { blood-forming organs and certain } \\
\text { disorders involving the immune } \\
\text { mechanism }\end{array}$} & \multirow{8}{*}{$\begin{array}{l}\text { Sanger } \\
\text { sequencing }\end{array}$} & \multirow{8}{*}{ [28] } \\
\hline & & & & $\begin{array}{c}\text { c.592 T > C } \\
\text { (p.Cys198Arg) }\end{array}$ & & & \\
\hline & & & & $\begin{array}{c}\text { c.1615 G > C } \\
\text { (p.Gly539Arg) }\end{array}$ & & & \\
\hline & & & & $\begin{array}{c}\text { c.2409 T > C } \\
\text { (p.Asn784Asn) }\end{array}$ & & & \\
\hline & & & & $\begin{array}{c}\text { c.2167 G > A } \\
\text { (p.Ala704Thr) }\end{array}$ & & & \\
\hline & & & & $\begin{array}{l}\text { c.3870-3871insA } \\
\text { (p.1271LysfsX29) }\end{array}$ & & & \\
\hline & & & & $\begin{array}{c}\text { c.1696 C > T } \\
\text { (p.Leu547Phe) }\end{array}$ & & & \\
\hline & & & & $\begin{array}{l}\text { c.1492 G > A } \\
\text { (p.Gly479Arg) }\end{array}$ & & & \\
\hline
\end{tabular}


Table 1. Cont.

\begin{tabular}{|c|c|c|c|c|c|c|c|}
\hline Disease Name & OMIM & Inheritance & Gene (OMIM) & Genetic Variant(s) & ICD-10 Classification & $\begin{array}{l}\text { Approach Used } \\
\text { for Molecular } \\
\text { Diagnosis }\end{array}$ & References \\
\hline & & & & c.77 T > C (p.Leu7Pro) & & & \\
\hline & & & & $\begin{array}{l}\text { c.5071-5075delATGAA } \\
(\mathrm{p} .1671-3 \mathrm{fs} X)\end{array}$ & & & \\
\hline & & & & $\begin{array}{c}\text { c.3637-3638delA } \\
\text { (p.1191IlefsX5) }\end{array}$ & & & \\
\hline & & & & $\begin{array}{l}\text { c.4379-4380insA } \\
\text { (p.1441LysfsX2) }\end{array}$ & & & \\
\hline & & & & $\begin{array}{c}\text { c.3637-3638insA } \\
\text { (p.1191LeufsX29) }\end{array}$ & & & \\
\hline & & & & $\begin{array}{l}\text { c.2236-2237insT } \\
\text { (p.727SerfsTer) }\end{array}$ & & & \\
\hline Gaucher disease type III A & 231000 & $\mathrm{AR}$ & GBA (606463) & $\begin{array}{l}\text { c.1330_1331delGAinsCC } \\
\text { (p.Asp444Pro) }\end{array}$ & $\begin{array}{l}\text { Endocrine, nutritional, and } \\
\text { metabolic diseases }\end{array}$ & $\begin{array}{l}\text { Sanger } \\
\text { sequencing }\end{array}$ & [29] \\
\hline $\begin{array}{l}\text { Junctional epidermolysis bullosa } \\
\text { (JEB) Herlitz type }\end{array}$ & 226700 & $\mathrm{AR}$ & $\begin{array}{l}\text { LAMA3 } \\
(600805)\end{array}$ & $\begin{array}{l}\text { c. } 2865 C \text { > G } \\
\text { (p.His955Gln) }\end{array}$ & $\begin{array}{c}\text { Congenital malformations, } \\
\text { deformations, and chromosomal } \\
\text { abnormalities }\end{array}$ & $\begin{array}{l}\text { Sanger } \\
\text { sequencing }\end{array}$ & {$[30]$} \\
\hline Lafora disease & 254780 & $\mathrm{AR}$ & $\begin{array}{l}\text { EPM2A } \\
(607566)\end{array}$ & $\begin{array}{c}\text { c.659 T > A } \\
\text { (p.Leu220Gln) }\end{array}$ & Diseases of the nervous system & $\begin{array}{c}\text { Sanger } \\
\text { sequencing }\end{array}$ & {$[31]$} \\
\hline
\end{tabular}


Table 1. Cont.

\begin{tabular}{|c|c|c|c|c|c|c|c|}
\hline Disease Name & OMIM & Inheritance & Gene (OMIM) & Genetic Variant(s) & ICD-10 Classification & $\begin{array}{l}\text { Approach Used } \\
\text { for Molecular } \\
\text { Diagnosis }\end{array}$ & References \\
\hline Lysosomal acid lipase deficiency & 278000 & $\mathrm{AR}$ & LIPA (613497) & c.153 C > A (p.Tyr51Ter) & $\begin{array}{l}\text { Endocrine, nutritional, and } \\
\text { metabolic diseases }\end{array}$ & $\begin{array}{c}\text { Sanger } \\
\text { sequencing }\end{array}$ & [32] \\
\hline $\begin{array}{l}\text { Maple syrup urine disease, type } \\
\qquad \mathrm{Ib}\end{array}$ & 248600 & $\mathrm{AR}$ & $\begin{array}{l}B C K D H B \\
(248611)\end{array}$ & $\begin{array}{c}\text { c.716A > G } \\
\text { (p.Glu239Gly) }\end{array}$ & $\begin{array}{l}\text { Endocrine, nutritional, and } \\
\text { metabolic diseases }\end{array}$ & $\begin{array}{c}\text { Sanger } \\
\text { sequencing }\end{array}$ & {$[33]$} \\
\hline Meckel syndrome type 2 & 603194 & $\mathrm{AR}$ & TMEM216(613277) & $\begin{array}{c}\text { c.341T > G } \\
\text { (p.Leu114Arg) }\end{array}$ & $\begin{array}{c}\text { Congenital malformations, } \\
\text { deformations, and chromosomal } \\
\text { abnormalities }\end{array}$ & $\begin{array}{c}\text { Exome } \\
\text { sequencing }\end{array}$ & [34] \\
\hline MELAS syndrome & 540000 & Mitochondrial & MT-TV (590105) & m.1640A > G & Diseases of the nervous system & $\begin{array}{c}\text { Sanger } \\
\text { sequencing }\end{array}$ & [35] \\
\hline Microphthalmia isolated, 6 & 613517 & $\mathrm{AR}$ & $\begin{array}{l}\text { PRSS56 } \\
(613858)\end{array}$ & $\begin{array}{c}\text { c.1059_1066insC } \\
\text { (p.Gln356ProfsTer152) }\end{array}$ & $\begin{array}{c}\text { Congenital malformations, } \\
\text { deformations, and chromosomal } \\
\text { abnormalities }\end{array}$ & $\begin{array}{c}\text { Sanger } \\
\text { sequencing }\end{array}$ & [36] \\
\hline Miyoshi muscular dystrophy 1 & 254130 & $\mathrm{AR}$ & DYSF (603009) & c. $4597-2 \mathrm{~A}>\mathrm{G}$ & Diseases of the nervous system & $\begin{array}{l}\text { Targeted Gene } \\
\text { Sequencing }\end{array}$ & [37] \\
\hline \multirow{5}{*}{ Mucopolysaccharidosis type II } & \multirow{5}{*}{309900} & \multirow{5}{*}{$\mathrm{XL}$} & \multirow{5}{*}{ IDS (300823) } & c.263G > A (p.Arg88Pro) & \multirow{5}{*}{$\begin{array}{l}\text { Endocrine, nutritional, and } \\
\text { metabolic diseases }\end{array}$} & \multirow{5}{*}{$\begin{array}{l}\text { Sanger } \\
\text { sequencing }\end{array}$} & \multirow{5}{*}[38]{} \\
\hline & & & & c.610C > T (p.Gln204Ter) & & & \\
\hline & & & & $\begin{array}{l}\text { c.1348G > A } \\
\text { (Asp450Asn) }\end{array}$ & & & \\
\hline & & & & c.281G > A (p.Gly94Asp) & & & \\
\hline & & & & $\begin{array}{c}\text { c.1186C > T } \\
\text { (p.Gln396Ter) }\end{array}$ & & & \\
\hline
\end{tabular}


Table 1. Cont

\begin{tabular}{|c|c|c|c|c|c|c|c|}
\hline Disease Name & OMIM & Inheritance & Gene (OMIM) & Genetic Variant(s) & ICD-10 Classification & $\begin{array}{c}\text { Approach Used } \\
\text { for Molecular } \\
\text { Diagnosis }\end{array}$ & References \\
\hline \multirow{7}{*}{$\begin{array}{c}\text { Mucopolysaccharidosis type } \\
\text { IIIA }\end{array}$} & \multirow{7}{*}{252900} & \multirow{7}{*}{$\mathrm{AR}$} & \multirow{7}{*}{ SGSH (605270) } & c.2t $>C$ (p.Met1Thr) & \multirow{7}{*}{$\begin{array}{l}\text { Endocrine, nutritional, and } \\
\text { metabolic diseases }\end{array}$} & \multirow{7}{*}{$\begin{array}{c}\text { Sanger } \\
\text { sequencing }\end{array}$} & \multirow{7}{*}{ [39] } \\
\hline & & & & $\begin{array}{c}\text { c. } 1129 \mathrm{C}>\mathrm{T} \\
\text { (p.Arg377Cys) }\end{array}$ & & & \\
\hline & & & & g.75802301_75809393del & & & \\
\hline & & & & $\begin{array}{l}\text { c.1093C > T } \\
\text { (p.Gln365Ter) }\end{array}$ & & & \\
\hline & & & & $\begin{array}{c}\text { c.29dup } \\
\text { (p.Leu11AlafsTer22) }\end{array}$ & & & \\
\hline & & & & c.197C > G (p.Ser66Trp) & & & \\
\hline & & & & $\begin{array}{c}\text { c.1080del } \\
\text { (p.Val361SerfsTer52) }\end{array}$ & & & \\
\hline \multirow[b]{2}{*}{ Mucopolysaccharidosis type IIIB } & \multirow[b]{2}{*}{252920} & \multirow[b]{2}{*}{ AR } & \multirow[b]{2}{*}{ NAGLU (60970) } & c.1674C > G (p.Tyr558X) & \multirow[b]{2}{*}{$\begin{array}{l}\text { Endocrine, nutritional, and } \\
\text { metabolic diseases }\end{array}$} & \multirow{2}{*}{$\begin{array}{l}\text { Sanger } \\
\text { sequencing }\end{array}$} & \multirow[b]{2}{*}{ [39] } \\
\hline & & & & $\begin{array}{c}\text { c.1811C > T } \\
\text { (p.Pro604Leu) }\end{array}$ & & & \\
\hline \multirow{2}{*}{$\begin{array}{c}\text { Mucopolysaccharidosis type } \\
\text { IIIC }\end{array}$} & \multirow[b]{2}{*}{252930} & \multirow[b]{2}{*}{$\mathrm{AR}$} & \multirow{2}{*}{$\begin{array}{r}\text { HGSNAT } \\
(610453)\end{array}$} & c.1209G > A (p.Trp403X) & \multirow{2}{*}{$\begin{array}{l}\text { Endocrine, nutritional, and } \\
\text { metabolic diseases }\end{array}$} & \multirow{2}{*}{$\begin{array}{l}\text { Sanger } \\
\text { sequencing }\end{array}$} & \multirow[b]{2}{*}{ [39] } \\
\hline & & & & $\begin{array}{l}\text { c.1880A > G } \\
\text { (p.Tyr627Cys) }\end{array}$ & & & \\
\hline Myoclonic epilepsy of Lafora & 254780 & AR & $\begin{array}{l}\text { EPM2A } \\
(607566)\end{array}$ & $\begin{array}{c}\text { c.659 T > A } \\
\text { (p.Leu220Gln) }\end{array}$ & $\begin{array}{l}\text { Diseases of the nervous } \\
\text { System }\end{array}$ & $\begin{array}{c}\text { Sanger } \\
\text { sequencing }\end{array}$ & [31] \\
\hline \multirow{2}{*}{ Nephropathic cystinosis } & \multirow{2}{*}{219800} & \multirow{2}{*}{ AR } & \multirow{2}{*}{ CTNS (606272) } & $\begin{array}{c}\text { c.1515G > A } \\
\text { (p.Gly308Arg) }\end{array}$ & \multirow{2}{*}{$\begin{array}{l}\text { Endocrine, nutritional, and } \\
\text { metabolic diseases }\end{array}$} & \multirow{2}{*}{$\begin{array}{c}\text { Sanger } \\
\text { sequencing }\end{array}$} & \multirow{2}{*}{ [40] } \\
\hline & & & & $\begin{array}{c}\text { c.771_793del } \\
\text { (p.Gly258SerfsTer30) }\end{array}$ & & & \\
\hline $\begin{array}{l}\text { Punctate palmoplantar } \\
\text { keratoderma type } 1\end{array}$ & 148600 & $\mathrm{AD}$ & $\begin{array}{l}A A G A B \\
(614888)\end{array}$ & c.481C > T (p.Arg161Ter) & $\begin{array}{c}\text { Congenital malformations, } \\
\text { deformations, and chromosomal } \\
\text { abnormalities }\end{array}$ & $\begin{array}{l}\text { Whole exome } \\
\text { sequencing }\end{array}$ & [41] \\
\hline
\end{tabular}




\subsection{Comorbidity among Tunisians}

Comorbidity is defined as the expression of another disease in addition to the primary disease. This phenomenon has been reported among Tunisian families (Romdhane et al., 2016) [42]. In our updated data, we have identified fourteen co-occurrences of multiple diseases since 2016 (Table 2). All of them belong to the genetic disease-genetic disease class of comorbid associations (in other words, both diseases are caused by a mutation in a single gene or multiple genes) with a consanguinity history among six of them. Both familial (6) and individual (8) comorbidities have been identified. They are either with the same mode of transmission or not. The comorbid associations include diseases of the same pathological group, for example, genodermatoses (Ichthyosis congenital and Erythrokeratodermia variabilis, Xeroderma pigmentosum group $\mathrm{C}$, and Rothmund Thomson syndrome) or not (Table 2).

Table 2. Comorbid associations described among Tunisians between 2016 and 2020.

\begin{tabular}{|c|c|c|c|c|}
\hline Associations & Familial/Individual & Inheritance & Consanguinity & References \\
\hline Allgrove syndrome-Hearing loss & Familial & $\mathrm{AR}-\mathrm{AR}$ & Yes & $\begin{array}{l}\text { Mkaouar et al., } \\
\text { unpublished }\end{array}$ \\
\hline $\begin{array}{l}\text { Amyotrophic lateral } \\
\text { sclerosis-Behcet's disease }\end{array}$ & Individual & AD, AR-Unknown & NA & [43] \\
\hline Autism-Hearing loss & Familial & Complex heredity-AR & Yes & $\begin{array}{l}\text { Lahbib et al., } \\
\text { unpublished }\end{array}$ \\
\hline Cutis laxa-Pulmonary disease & Familial & Unknown-Unknown & NA & $\begin{array}{l}\text { Tinsa et al., } \\
\text { unpublished }\end{array}$ \\
\hline $\begin{array}{c}\text { Growth hormone } \\
\text { deficiency-Immunodeficiency }\end{array}$ & Individual & Unknown-Unknown & NA & $\begin{array}{l}\text { Tinsa et al., } \\
\text { unpublished }\end{array}$ \\
\hline $\begin{array}{c}\text { Ichthyosis congenital autosomal } \\
\text { recessive } 1 \text {-Erythrokeratodermia } \\
\text { variabilis }\end{array}$ & Familial & $\mathrm{AR}-\mathrm{AD}$ & No & $\begin{array}{l}\text { Laroussi et al., } \\
\text { Unpublished }\end{array}$ \\
\hline $\begin{array}{l}\text { Ichthyosis congenital autosomal } \\
\text { recessive 5-hearing loss }\end{array}$ & Individual & $\mathrm{AR}-\mathrm{AR}$ & Yes & {$[44]$} \\
\hline $\begin{array}{l}\text { Ichthyosis congenital autosomal } \\
\text { recessive 1-Muscular dystrophy limb } \\
\text { girdle type 2A }\end{array}$ & Individual & AR-AR & Yes & $\begin{array}{l}\text { Mezzi et al., } \\
\text { Unpublished }\end{array}$ \\
\hline $\begin{array}{l}\text { Incontinentia pigmenti-Noonan } \\
\text { syndrome }\end{array}$ & Individual & $\mathrm{XLD}-\mathrm{AD}$ & No & {$[45]$} \\
\hline $\begin{array}{c}\text { Maternally inherited } \\
\text { diabetes-deafness-Retinopathy }\end{array}$ & Individual & $\begin{array}{l}\text { Mitochondrial- } \\
\text { Unknown }\end{array}$ & No & {$[46]$} \\
\hline $\begin{array}{l}\text { Niemann-Pick disease type } \\
\text { B-Systemic lupus erythematous }\end{array}$ & Familial & $\mathrm{AR}-\mathrm{AD}$ & No & [47] \\
\hline $\begin{array}{c}\text { Pernicious } \\
\text { anemia-Pseudohypoparathyroidism }\end{array}$ & Individual & Unknown-Unknown & NA & $\begin{array}{l}\text { Tinsa et al., } \\
\text { unpublished }\end{array}$ \\
\hline $\begin{array}{l}\text { Xeroderma pigmentosum group } \\
\text { A-Autoimmune polyendocrinopathy } \\
\text { syndrome I }\end{array}$ & Individual & $\mathrm{AR}-\mathrm{AR}, \mathrm{AD}$ & Yes & $\begin{array}{l}\text { Messaoud et al., } \\
\text { Unpublished }\end{array}$ \\
\hline $\begin{array}{l}\text { Xeroderma pigmentosum group } \\
\text { C-Rothmund Thomson syndrome }\end{array}$ & Familial & $\mathrm{AR}-\mathrm{AR}$ & Yes & $\begin{array}{l}\text { Ezzine et al., } \\
\text { Unpublished }\end{array}$ \\
\hline
\end{tabular}




\subsection{Phenotypic Features of Genetic Diseases in Tunisia}

Atypical phenotypes are also reported and characterize several genetic diseases among Tunisians. By atypical clinical presentations, we mean unusual symptoms of the disease. Chanarin-Dorfman syndrome, palmoplantar keratoderma, juvenile Parkinson's disease, and multiple endocrine neoplasia type $2 \mathrm{~A}$ were described in Tunisian patients with unusual presentations (Table 3).

Table 3. Genetic diseases with unusual clinical findings among Tunisians.

\begin{tabular}{|c|c|c|c|}
\hline Diseases & Unusual Clinical Findings & Case count & References \\
\hline $\begin{array}{c}\text { Chanarin-Dorfman } \\
\text { syndrome }\end{array}$ & Thyroid function impairment & 7 & [48] \\
\hline $\begin{array}{l}\text { Palmoplantar } \\
\text { keratoderma }\end{array}$ & $\begin{array}{l}\text { Abnormal cornification and a diffuse } \\
\text { yellowish keratoderma with the } \\
\text { characteristic skin thickening }\end{array}$ & 1 & [49] \\
\hline $\begin{array}{c}\text { Juvenile Parkinson } \\
\text { disease }\end{array}$ & $\begin{array}{l}\text { No evidence of sleep or autonomic } \\
\text { dysfunctions and psychiatric } \\
\text { disorders in both patients }\end{array}$ & 1 & [50] \\
\hline
\end{tabular}

\subsection{Epidemiology of Genetic Diseases in Tunisia}

For epidemiological parameters, we focused on the frequency of the whole reported disease spectrum by targeting either the prevalence or the incidence. We noticed the paucity of such epidemiological data as only $26(4.6 \%)$ genetic diseases described among Tunisians have defined prevalence and/or incidence (Table 4). For some genetic disorders (five diseases), the epidemiological data are described among specific ethnic groups or in specific regions. For the remaining diseases, only the number of studied patients and/or families is reported. In an attempt to compare these epidemiological data, we tried to use the same scale of a rare disease threshold prevalence in Europe (1/2000). By aligning to this definition, we found that seven diseases largely exceed this threshold. For ten others, the available epidemiological data suggests that they are ultra-rare diseases (Table 4).

\subsection{Current Situation of Newborn and/or Carrier Screening for Genetic Diseases in Tunisia}

On 1 July 2021, the second PubMed-MeSH query described above yielded 732 results, of which 26 articles have been retained for further analysis. Three articles (in French) found in Google Scholar have also been included. Ultimately 29 articles were screened. In all of these studies, the authors highlight the key role of screening for genetic diseases as a state-run health program in Tunisia. However, neonatal newborn and carrier screenings were limited to pilot experiments for only six conditions, and they have not been replicated or extended geographically to the level of the country. Systematic newborn screening for hearing impairment is exclusively conducted in the governorate of Sfax in the south (Table 5). In fact, 16 disorders are likely frequent among Tunisians, of which 10 are part of the Recommended Uniform Screening Panel. All of them are associated with founder and/or recurrent mutations, and 14 met all the 7 ACOG criteria, with 2 late-onset diseases (Table S2 Supplementary data). 
Table 4. Genetic diseases with available epidemiological data among Tunisians.

\begin{tabular}{|c|c|c|c|c|c|}
\hline Genetic Disease (MIM) & Frequency & References & $\begin{array}{l}\text { Measure of } \\
\text { Estimation }\end{array}$ & State/Region/Group & Prevalence in Orphanet ** \\
\hline $\begin{array}{c}\text { Anemia, Nonspherocytic hemolytic, due to } \\
\text { G6PD deficiency (ANH- G6PD) } \\
\text { (300908) }\end{array}$ & $18,400 /$ million/year & [51] & Incidence & All across Tunisia & $<1 / 1,000,000$ \\
\hline$\beta$ thalassemia ( $\beta$-thal) (613985) & $44.2 / 2000$ & [52] & Prevalence & All across Tunisia & $1-9 / 1,000,000$ \\
\hline Creutzleldt-Jakob Disease (CJD*) (123400) & $2.3 /$ million/year & [53] & Incidence & Among Tunisian Jews & $<1 / 1,000,000$ \\
\hline \multirow[b]{2}{*}{ Cystic fibrosis $\left(\mathrm{CF}^{*}\right)(219700)$} & $\begin{array}{c}1.5 \text { new cases/year } \\
0.4 / 1000\end{array}$ & \multirow[b]{2}{*}[54]{} & \multirow{2}{*}{$\begin{array}{l}\text { Incidence } \\
\text { Prevalence }\end{array}$} & \multirow{2}{*}{$\begin{array}{l}\text { In the Pediatric department B of the } \\
\text { Children's Hospital Béchir Hamza de } \\
\text { Tunis (among patients' series) } \\
\text { Most from the north and the south } \\
\text { of Tunisia }\end{array}$} & NA \\
\hline & $\begin{array}{c}0.4 / 1000 \\
\text { Its prevalence was } 0.4 \text { per } \\
1000 \text { hospitalizations. }\end{array}$ & & & & $1-9 / 100,000$ \\
\hline Dermatitis, Atopic (ATOD) (603165) & $\begin{array}{l}451 \text { cases during a } 7 \text { years } \\
\text { period }\end{array}$ & [55] & Incidence & All across Tunisia & NA \\
\hline $\begin{array}{l}\text { Epidermolysis bullosa dystrophica } \\
\text { Hallopeau-Simens type } 1 \text { (RDEB*) (226600) }\end{array}$ & $2.3 / 2000$ & $\begin{array}{c}\text { Cherif et al., } 2005 \\
\text { unpublished }\end{array}$ & Prevalence & All across Tunisia & $<1 / 1,000,000$ \\
\hline Exfoliation syndrome (XFS) (177650) & $220 / 2000$ & [57] & Prevalence & All across Tunisia & NA \\
\hline $\begin{array}{l}\text { Familial Adenomatous polyposis of the } \\
\text { colon (FAP1) (MIM: 175100) }\end{array}$ & 74/million/year & [58] & Incidence & All across Tunisia & $1-9 / 100,000$ \\
\hline $\begin{array}{l}\text { Fanconi anemia (FA) } \\
\qquad(227650)\end{array}$ & 1.4/million/year & [59] & Incidence & All across Tunisia & $1 / 160,000$ \\
\hline Familial hypercholesterolemia, (FHCL*) & $12.12 / 2000$ & {$[60]$} & Prevalence & In central and southern Tunisia & $1-9 / 1,000,000$ \\
\hline Gaucher disease, type I (GD1) (230800) & $0.0096 / 2000$ & [61] & Prevalence & All across Tunisia & $1 / 100,000$ \\
\hline Glycine encephalopathy (GCE*) (605899) & $1 / 9322$ & [62] & Incidence & In the governorate of Kairouan & $1-9 / 1,000,000$ \\
\hline \multirow{2}{*}{$\begin{array}{c}\text { Glycogen storage disease type Ia (GSD1A*) } \\
(232200)\end{array}$} & $0.02 / 2000$ & \multirow{2}{*}{ [63] } & Prevalence & \multirow{2}{*}{ In the north of Tunisia } & NA \\
\hline & 7.93/million/year & & Incidence & & 1/100,000 (Incidence) \\
\hline Hemoglobinopathies & $89.6 / 2000$ & [52] & Prevalence & All across Tunisia & NA \\
\hline Hurler syndrome $\left(\mathrm{HS}^{*}\right)$ (607014) & $0.064 / 2000$ & [64] & Prevalence & In Tunisian Jews & $1 / 200,000$ \\
\hline
\end{tabular}


Table 4. Cont

\begin{tabular}{|c|c|c|c|c|c|}
\hline Genetic Disease (MIM) & Frequency & References & $\begin{array}{l}\text { Measure of } \\
\text { Estimation }\end{array}$ & State/Region/Group & Prevalence in Orphanet ** \\
\hline $\begin{array}{l}\text { Limb-girdle Muscular dystrophytype 2C } \\
\text { (LGMD2C) (253700) }\end{array}$ & $0.6 / 2000$ & {$[65]$} & Prevalence & All across Tunisia & $1-9 / 1,000,000$ \\
\hline Lynch syndrome 1 (LS1) (120435) & 70/million/year & [66] & Incidence & All across Tunisia & NA \\
\hline Megaloblastic anemia $1\left(\right.$ MGA1*) $^{*}(261100)$ & $2 / 2000$ & [67] & Prevalence & In Tunisian Jews & NA \\
\hline Mucopolysaccharidosis I (MPS1-S) (607016) & $0.0126 / 2000$ & [59] & Prevalence & All across Tunisia & $1 / 100,000$ \\
\hline $\begin{array}{l}\text { Mucopolysaccharidosis type IVA (MPS4A) } \\
(253000)\end{array}$ & $0.025 / 2000$ & [68] & Prevalence & All across Tunisia & $1-5 / 10,000$ \\
\hline $\begin{array}{c}\text { Mucopolysaccharidosis type VI (MPS 6) } \\
(253200)\end{array}$ & $0.013 / 2000$ & [62] & Prevalence & All across Tunisia & $1-9 / 1,000,000$ \\
\hline Niemann Pick disease B (607616) & $0.1 / 2000$ & [66] & Prevalence & & $1-9 / 1,000,000$ \\
\hline Phenylketonuria (PKU) (261600) & $\begin{array}{c}\text { Varies between } 0.29 / 2000 \\
\text { and } 0.6 / 2000\end{array}$ & [67] & Prevalence & All across Tunisia & $1-5 / 10,000$ \\
\hline Sickle Cell Anemia (SCA) (603903) & $37.8 / 2000$ & [52] & Prevalence & All across Tunisia & $1 / 150$ \\
\hline $\begin{array}{l}\text { Xeroderma pigmentosum, complementation } \\
\text { group A (XPA) (278700) }\end{array}$ & $0.2 / 2000$ & [69] & Prevalence & All across Tunisia & $1 / 1,000,000$ \\
\hline
\end{tabular}

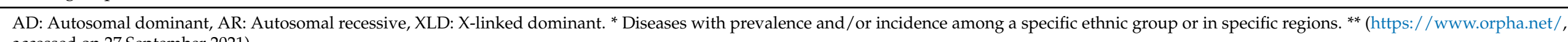
accessed on 27 September 2021). 
Table 5. Screening of genetic diseases in Tunisia.

\begin{tabular}{|c|c|c|c|c|}
\hline Diseases (MIM) & Screening Type & Screening Techniques & Country Coverage & References \\
\hline$\alpha$-thalassemia & Prenatal screening & $\begin{array}{l}\text { DNA analysis from amniotic } \\
\text { fluid }\end{array}$ & $\begin{array}{l}\text { Biochemistry and molecular } \\
\text { biology department in } \\
\text { children's hospital of Tunis } \\
\text { (pilot study) }\end{array}$ & [70] \\
\hline$\beta$-thalassemia & $\begin{array}{l}\text { Prenatal screening } \\
\text { Carrier screening }\end{array}$ & $\begin{array}{c}\text { DNA analysis from amniotic } \\
\text { fluid } \\
\text { Screening for mutations } \\
\text { described in Tunisians }\end{array}$ & $\begin{array}{l}\text { Biochemistry and molecular } \\
\text { biology department in } \\
\text { children's hospital of Tunis } \\
\text { (pilot study) }\end{array}$ & [70] \\
\hline $\begin{array}{l}\text { Congenital } \\
\text { hypothyroidism }\end{array}$ & Newborn screening & $\begin{array}{l}\text { TSH and T4 } \\
\text { radioimmunoassay } \\
\text { on drops of blood }\end{array}$ & $\begin{array}{c}\text { Maternity and Neonatal } \\
\text { Centre in Tunis (pilot study) }\end{array}$ & Elkadri et al. * \\
\hline Cystic fibrosis & Prenatal screening & $\begin{array}{l}\text { Genetic analysis by denaturant } \\
\text { gradient gel electrophoresis } \\
\text { and denaturing high-pressure } \\
\text { liquid phase chromatography }\end{array}$ & $\begin{array}{l}\text { Biochemistry Laboratory, } \\
\text { Bechir Hamza Children's } \\
\text { hospital in Tunis } \\
\text { Center of maternity and } \\
\text { neonatology, in Tunis } \\
\text { (pilot study) }\end{array}$ & {$[71]$} \\
\hline $\begin{array}{l}\text { Hearing } \\
\text { impairment }\end{array}$ & Newborn screening & $\begin{array}{l}\text { Evoked otoacoustic emissions } \\
\text { (EOAE) and auditory brain } \\
\text { stem response (ABR) }\end{array}$ & $\begin{array}{l}\text { Charles Nicolle hospital of } \\
\text { Tunis ((pilot study) } \\
\text { Regional hospital in Nabeul } \\
\text { (pilot study) } \\
\text { Regional hospital in Sfax } \\
\text { (systematic) }\end{array}$ & $\begin{array}{l}\text { [72] } \\
\text { Feedback from } \\
\text { collaborating } \\
\text { clinician }\end{array}$ \\
\hline G6PD deficiency & Newborn screening & $\begin{array}{l}\text { Dosage of the enzymatic } \\
\text { activity using } \\
\text { spectrophotometric method }\end{array}$ & $\begin{array}{c}\text { Maternity and Neonatal } \\
\text { Centre in Tunis (pilot study) }\end{array}$ & [73] \\
\hline Phenylketonuria & Newborn screening & $\begin{array}{l}\text { Dosage of phenylalanine in } \\
\text { dried blood spots }\end{array}$ & $\begin{array}{l}\text { Hospital of La Rabta in Tunis } \\
\text { (pilot study) }\end{array}$ & [68] \\
\hline Sickle cell disease & Newborn screening & Isoelectrofocusing & $\begin{array}{l}\text { Maternity Centre of Aziza } \\
\text { Othmana Hospital and } \\
\text { Neonatal and Maternity } \\
\text { Centre_La Rabta in Tunis } \\
\text { (pilot study) }\end{array}$ & {$[74]$} \\
\hline
\end{tabular}

* Available online: http:/ / docplayer.fr/66862824-Depistage-de-1-hypothyroidie-congenitale-a-tunis-seuils-de-rappel-et-protocole.html (accessed on 27 September 2021).

\section{Discussion}

In this study, we attempted to provide a comprehensive and up-to-date overview of the genetic disease spectrum described among Tunisians. Our findings showed that the number of reported genetic conditions increased from 346 to 589 in the last eleven years (2010-2021). This highlights remarkable progress in the availability of molecular diagnosis of genetic diseases in Tunisia. The development of biomedical health research activities, the building capacities in this field, and the use of high-throughput sequencing technologies facilitated by international collaborations, have played a critical role in this progress [7].

Genetic disorders present a health burden, especially among inbred populations, including Arab countries and several African communities [9,75,76]. Here, in the recent spectrum, most of the genetic diseases described among Tunisians are autosomal recessive (59.8\%). A slight reduction is noticed compared to data reported in 2011; however, their frequency is still high, which could be explained by the socio-cultural features in the Tunisian population [19]. Indeed, consanguineous marriages, with an extreme predominance of first-cousin marriages associated with large family sizes, are responsible for the 
high prevalence of recessively inherited conditions among Tunisians $[20,22,23,77]$. It has been proven that there is an increased risk of almost eight times for expressing a recessive disease among first-cousin marriage's progeny [22].

Another impact of the elevated rate of consanguinity and inbreeding is the cooccurrence of two or more phenotypes within the same family [42]. Comorbidity, described particularly in inbred populations, makes genetic counseling and prenatal diagnosis challenging [42,44]. The study of Romdhane and her colleagues revealed 75 genetic disease co-occurrences identified in Tunisian patients. Thirty-nine (39) of them have been reported among Tunisian consanguineous families [42]. Since then, 14 additional comorbidities of genetic diseases in Tunisians were reported in our study, for which NGS techniques had largely contributed to the molecular diagnosis. Consanguinity was noted in six of them.

Several variants, either in the same gene or in related genes, were correlated with the high frequency of genetic diseases in inbred communities [76]. In the current report, we have found that the Tunisian mutational spectrum includes more than 896 mutations affecting 313 genes. Among these mutated genes, allelic heterogeneity was identified for 154 of them. Such characteristics render the diagnosis difficult and could lead to misdiagnosis, especially in developing countries, such as Tunisia $[7,10]$. Screening for founder mutations among endogamous populations is a cost-effective molecular diagnosis strategy [10], but it may be inefficient when applied to atypical rare phenotypes and comorbidities (Mezzi et al., Unpublished). Consequently, whole-exome sequencing (WES), could be an alternative choice to elucidate the molecular etiology of such conditions and is always recommended, especially when targeted mutation screening and/or targeted gene sequencing results are negative or ambiguous [78,79]. In Tunisia, molecular investigation of genetic diseases is mainly conducted using Sanger DNA sequencing. However, since 2013, we note that next-generation sequencing (NGS) has become more affordable to the scientific community, mainly through international collaborations. We found that the use of high-throughput sequencing has led to the identification of the molecular bases of at least 45 mendelian diseases described in Tunisians.

Autosomal dominant diseases were found in $24.5 \%$ of reported Tunisian patients. Although intrafamilial marriages have no important effect on the prevalence of autosomal dominant diseases, significant consequences on the expression of such disorders could not be completely excluded [7]. Indeed, consanguineous unions could increase the likelihood of homozygosity for dominant genetic variations $[20,80]$, which could induce more severe phenotypes and even atypical ones $[20,80]$. This was the case for a large consanguineous Tunisian family with granular corneal dystrophy type I in which homozygous members for a TGFBI mutation were the severely most affected [81]. Allelic comorbidity involving a dominant disease illustrated by the consanguineous family reported by Sfar et al., is also an excellent example of such an effect [82]. The parents, who presented a mild phenotype of neonatal hyperparathyroidism, were heterozygous for a CASR gene mutation. Their daughter had a severe hyperparathyroidism phenotype because of her homozygous state for the same mutation [82].

Accurate prevalence and incidence rates are crucial to developing an understanding of a disease's natural history and population burden [83]. However, among the 589 genetic disorders described among Tunisians, only $26(4.6 \%)$ have a defined prevalence and/or incidence yet without systematic updates. Some genetic diseases, including familial hypercholesterolemia $(12.12 / 2000)$ and hemoglobinopathies $(89.6 / 2000)$, are particularly frequent in Tunisia compared to Europe, where they are reported as rare diseases. Several others are rare and even ultra-rare, depending on the defined threshold. The scarcity of accurate epidemiological data could lead to misdiagnosis [84] and indicates the necessity of comprehensive epidemiology and patient registry creation $[7,20,85]$. Thus far, only three patient registries have been set for genetic disorders in Tunisia, namely, Gaucher Disease (http:/ / www.maladie-gaucher-tunisie.org/, accessed on 24 September 2021), Fanconi Anemia (http:/ / fanconi-tunisie.net/, accessed on 24 September 2021), and bleeding disorders [86]. Furthermore, they are not systematically updated, and they are facing 
personal patient data security problems and storage systems issues. Building rare genetic diseases registries in developing countries is challenging $[84,86]$. The main obstacle is the drastic lack of well-defined policies and the paucity of common protocols for these registries [84]. Moreover, data on rare genetic diseases are usually unharmonized (archived in hand-written form or in simple excel files). Consequently, data treatment and handling are time-consuming tasks [85]. In addition, the funding needed for the infrastructure of these registries could be challenging, as not all countries can cover their costs [85]. Considering the fact that epidemiological studies of rare and extremely rare diseases are difficult to set at the level of the country (as for classical epidemiological studies), we highly recommend national registries to establish either specific to each disease or to a group of diseases in order to have not only a better estimation of their figures but also for better care interventions. Moreover, developing a population-specific genetic diseases database is undoubtedly needed to have a complete overview of their spectrum in a given population. This informative online database will be a hopeful starting point for healthcare experts and researchers [87].

Molecular data availability on relatively frequent genetic rare diseases in inbred populations could be an ideal way to boost current research focusing on targeted therapies, including gene and mutation-specific therapies, hence leading to the implementation of precision medicine. Several diseases identified in the Tunisian population are subject to targeted therapy based on their genetic information. Bloom syndrome (BS) is a DNArepair disease characterized by a high risk of cancer at an early age [88]. It has been established that a correct diagnosis should be clearly set before using chemotherapy in BS patients because drug dose is highly dependent on the disease severity degree [88] while conventional chemo or radiotherapy would lead to a profound genomic instability and, consequently, enhanced carcinogenesis. In the same way, knowing the gene or the mutation is also fundamental for implementing efficient therapy. Indeed, improved cochlear implant outcomes have been observed among deaf Tunisian individuals for whom the hearing loss is related to GJB2 mutations and who were implanted at an early age [89,90]. Drugs targeting mutations are an innovative therapeutic approach in cystic fibrosis (CF) [91,92]. $\mathrm{CF}$ is a common genetic life-shortening condition in the Caucasian population, and it is not so rare in North Africa [93]. The CFTR gene has accumulated multiple founder mutations that could be used as a target for personalized therapy [94]. Lumacaftor was tested as an effective mutation-specific therapy for African-American CF patients targeting the shared founder mutation that is also reported in the Tunisian population [95]. Gene therapy is a promising strategy for the permanent treatment of genetic diseases. LGMD2C has the highest incidence in North Africa, and it is caused by the predominant founder deletion c.521delT in the SGCE gene [96,97]. A gene therapy trial has been performed targeting patients with these founder mutations as a crucial inclusion criterion [98]. Nine Tunisian patients were involved in this trial [98]. With mutation-specific therapies being under development, a correct diagnosis is more than ever mandatory for assessing whether patients are eligible or not for adequate treatments.

The increased focus on genetic diseases in Tunisia over the last decades has been spurred mainly by the clinical and molecular investigations intended to improve the diagnosis. Nevertheless, genetic services are lacking and are not equally distributed across the country. Indeed, only three main medical genetic services are available: Charles Nicolle hospital in Tunis, Farhat Hached hospital in Sousse, and Habib bourguiba hospital in Sfax. A unit for congenital and inherited diseases was recently built at the "Mongi Slim hospital" in La Marsa, providing cytogenetic tests. Furthermore, breast and cervical cancer screening has been integrated into basic health care services within the framework of national cancer control plans (Ministère de la Santé, Direction de Santé, Plan pour la lutte contre le cancer 2015-2019) (Tunis: Ministère de la Santé, 2015). In addition, the establishment of an NGS platform in Institut Pasteur de Tunis, allowed the setting up of molecular diagnosis of familial cancers, thus contributing to the better characterization and widening of the mutation spectrum of BRCA1/2 genes in the Tunisian population [99]. The increased prevalence 
of cystic fibrosis (CF) among Tunisian children and the potentially severe complications associated with this disease justify the management of $\mathrm{CF}$ by a multidisciplinary team of clinicians in the child pulmonology referral unit in Bechir Hamza children's Hospital of Tunis [54]. In this unit, englobing the largest national series, patients are managed by several therapeutic strategies (respiratory physiotherapy, antibiotherapy, aerosol therapy, vitamin therapy, inhaled corticosteroids, etc.) [54,94]. Genetic counseling and prenatal diagnosis are also proposed to families [54]. This example illustrates the importance of the referral centers for better knowledge of the disease where adequate clinical and molecular competencies are available, but it is still insufficient as the commitment of the national authorities is required in order to support these centers by providing adequate technological platforms.

From a public health perspective, experts and policymakers have opted that newborn and carrier screening should be conducted as a state-run program [100,101]. This will allow early detection of genetic conditions and congenital disorders, leading to reduced health disparities and mortality at a young age $[102,103]$. The infant mortality rate is an indicator of a state's health, and reducing this rate is an explicit goal of sustainable development (2030 United Nations Sustainable Development Goals). Several genetic conditions are recommended to be screened among newborns and/or at-risk individuals around the world. Among these, we have the Recommended Uniform Screening Panel (RUSP) issued by the U.S. Health and Human Services (HSS) Federal Advisory Committee on Heritable Disorders in Newborns and Children (ACHDNC). The RUSP includes 35 core conditions (for which newborn screening is highly recommended) and 26 secondary conditions (for which newborn screening is optional). Furthermore, a data-driven evaluation has been conducted in which an Expanded Carrier Screening (ECS) panel of 172 conditions have been defined and met all the required criteria defined by the American College of Obstetricians and Gynecologists $[104,105]$. In the MENA region, reducing genetic condition expression, particularly those recessively inherited, is a salient issue mainly due to their high prevalence because of inbreeding and the severe expression in newborns [106,107]. However, among several countries of this region, newborn screening is not introduced systematically as a health policy, and it is only limited to pilot projects [106,108]. In fact, only Gulf states have a newborn screening panel for 20 metabolic conditions [106,109]. Egypt and Palestine also have a national neonatal screening plan for congenital hypothyroidism and phenylketonuria [110-112]. Of the disorders recommended to be screened (RUSP and ECS panel), $49 \%$ are described among Tunisian patients (Table S3 Supplementary Data), representing $19 \%$ of the genetic disease spectrum in Tunisia and could be considered, therefore, as a potential target for intervention. The absence of such an intervention in Tunisia could be explained by the inability of the Ministry of Health and of the social security system to ensure adequate care or health coverage for individuals screened as positive $[108,113]$. Consequently, we propose, instead of systematic neonatal screening, to conduct cascade carrier screening with specific regional programs that take into account the most frequent diseases and underlying deleterious founder alleles. This helps to decrease morbidity and mortality, as well as to improve the quality of life and life expectancy for patients with genetic diseases.

A comprehensive genetic education and premarital genetic counseling programs can also help to reduce the burden of genetic diseases in inbred communities [114,115]. In Bahrain, educational programs aimed at high school children have had a marked beneficial effect in lessening the incidence of sickle cell disease [116]. It has been suggested that raising public awareness of sickle cell disease through prevention campaigns using informational programs with different communication media would be efficient to reduce the burden of the disease in the population in addition to programs that have proven their efficiencies, such as student screening programs, premarital counseling, and newborn screening [117].

Patient support groups could act as key support for rare genetic diseases. As stated earlier, hearing loss constitutes a frequent disease group in our population. In an attempt to improve the auditory health in the Tunisian population, hearing screening and awareness- 
raising campaigns are being organized by NGOs, such as ICHARA, in the frame of a science shop project participatory research support [118].

\section{Conclusions}

Our study provides a better knowledge of the genetic diseases spectrum in Tunisia, which is useful for this country, as well as for neighboring countries, in order to improve the diagnosis, to reduce misdiagnosis, and therefore, patients will have earlier and better management. This would also help to avoid possible complications and reduce the costs of managing these diseases. Molecular and genetic data for these diseases are becoming more widely available through the development of biomedical health research activities and access to high-throughput next-generation sequencing. However, the Tunisian population still faces challenges, especially in the case of dual diagnosis and co-occurrence in the same patient or family, which delays the appropriate patient management. Taking this into account, in addition to the relatively high genetic and mutational heterogeneity, we recommend the creation of diagnostic referral centers that leverage some technologies, such as whole-exome sequencing, as a first intention tool for genetic disease elucidation in the country. These specialized structures could potentially offer accurate molecular diagnostic services at a lower cost and allow faster, efficient patient management. Furthermore, patient management should include multidisciplinary team investigators working in a well-defined care pathway. Vigilant monitoring of emerging clinical manifestations is recommended, especially in the case of genetic disorders. Furthermore, studies are needed to accurately set the epidemiological data of genetic diseases aiming to implement better and appropriate healthcare measures. Patient support groups have a significant role to play in promoting patient voice and influencing the decision-makers.

Supplementary Materials: The following are available online at https://www.mdpi.com/article/ 10.3390/genes12111820/s1, Table S1: Mutated genes among Tunisians, Table S2: Frequent genetic diseases in Tunisia, Table S3: Genetic diseases screened recommended for newborn and/or carrier screening.

Author Contributions: Conceptualization: N.M., L.R. and S.A. Methodology/literature search: N.M. and L.R. Data collection: N.M., O.M., R.M. (Rahma Mkaouar), N.Z., S.R., G.A., C.C., F.M., I.O., Y.H., A.Z., R.M. (Ridha Mrad), S.A. and L.R. Data analysis: N.M. Validation: L.R., O.M., S.A. and R.M. (Ridha Mrad), Writing_original draft preparation: N.M. Writing—review and editing: N.M., O.M., R.M. (Rahma Mkaouar), N.Z., G.A., C.C., F.M., I.O., A.Z., R.M. (Ridha Mrad), S.A. and L.R. All authors have read and agreed to the published version of the manuscript.

Funding: This work was supported by the Tunisian Ministry of Public Health (Personal costs), the Ministry of Higher Education and Scientific Research (LR20IPT05), and the project of young researchers' promotion for their contributions to this study (19PEJC06-07). N.M. is a recipient of a MOBIDOC fellowship, funded by The Ministry of Higher Education and Scientific Research through the PromEssE project and managed by the ANPR. The funders had no role in study design, data collection, and analysis, decision to publish, or preparation of the manuscript.

Institutional Review Board Statement: Not applicable.

Informed Consent Statement: Not applicable.

Data Availability Statement: Not applicable.

Acknowledgments: The authors are grateful to clinicians' collaborators for sharing their field experience and feedback that was of great value in our study.

Conflicts of Interest: The authors declare that they have no conflict of interest regarding this study.

\section{References}

1. Ferreira, C.R. The burden of rare diseases. Am. J. Med. Genet. Part A 2018, 179, 885-892. [CrossRef]

2. Thomas, S.; Caplan, A. The Orphan Drug Act Revisited. JAMA 2019, 321, 833-834. [CrossRef]

3. Richter, T.; Nestler-Parr, S.; Babela, R.; Khan, Z.M.; Tesoro, T.; Molsen, E.; Hughes, D.A. Rare Disease Terminology and DefinitionsA Systematic Global Review: Report of the ISPOR Rare Disease Special Interest Group. Value Health 2015, 18, 906-914. [CrossRef] 
4. Sara Cannizzo, V.L.; Palla, I.; Pirri, S.; Trieste, L.; Triulzi, I.; Turchetti, G. Rare diseases under different levels of economic analysis: Current activities, challenges and perspectives. RMD Open 2018, 4 (Suppl. 1), e000794. [CrossRef]

5. Luo, E.; Liu, H.; Zhao, Q.; Shi, B.; Chen, Q. Dental-craniofacial manifestation and treatment of rare diseases. Int. J. Oral. Sci. 2019, 11, 9. [CrossRef] [PubMed]

6. Lihadh Al-Gazali, H.H.; Al-Arrayad, S. Genetic disorders in the Arab world. Br. Med. J. 2006, 333, 831-834. [CrossRef] [PubMed]

7. Romdhane, L.; Mezzi, N.; Hamdi, Y.; El-Kamah, G.; Barakat, A.; Abdelhak, S. Consanguinity and inbreeding in health and disease in North African populations. Annu. Rev. Genom. Hum. Genet. 2019, 20, 155-179. [CrossRef] [PubMed]

8. Zayed, H. The Arab genome: Health and wealth. Gene 2016, 592, 239-243. [CrossRef] [PubMed]

9. Tadmouri, G.O.; Nair, P.; Obeid, T.; Al Ali, M.T.; Al Khaja, N.; Hamamy, H.A. Consanguinity and reproductive health among Arabs. J. Reprod. Health 2009, 6, 17. [CrossRef] [PubMed]

10. Romdhane, L.; Kefi, R.; Azaiez, H.; Ben Halim, N.; Dellagi, K.; Abdelhak, S. Founder mutations in Tunisia: Implications for diagnosis in North Africa and Middle East. Orphanet J. Rare Dis. 2012, 7, 52. [CrossRef]

11. Frigi, S.; Cherni, L.; Fadhlaoui-Zid, K.; Benammar-Elgaaied, A. Ancient local evolution of African mtDNA haplogroups in Tunisian Berber populations. Hum. Biol. 2010, 82, 367-384. [CrossRef] [PubMed]

12. Kefi, R.; Hsouna, S.; Ben Halim, N.; Lasram, K.; Romdhane, L.; Messai, H.; Abdelhak, S. Phylogeny and genetic structure of Tunisians and their position within Mediterranean populations. Mitochondrial DNA 2015, 26, 593-604. [CrossRef]

13. Mughal, M. In Tunisia, in Native Peoples of the World: An Encyclopedia of Groups, Cultures, and Contemporary Issues; Routledge: New York, NY, USA, 2013; pp. 688-689.

14. Hajjej, A.; Almawi, W.Y.; Hattab, L.; El-Gaaied, A.; Hmida, S. HLA Class I and Class II Alleles and Haplotypes Confirm the Berber Origin of the Present Day Tunisian Population. PLoS ONE 2015, 10, e0136909. [CrossRef] [PubMed]

15. Ben Romdhane, H.; Tlili, F.; Skhiri, A.; Zaman, S.; Phillimore, P. Health system challenges of NCDs in Tunisia. Int. J. Public Health 2015, 60, 39-46. [CrossRef] [PubMed]

16. Arfa, C.; Achouri, H. Tunisia: "Good Practice" in expanding health care coverage: Lessons from reforms in a country in transition. In Good Practices in Health Financing Lessons from Reforms in Low-and Middle-Income Countries; The World Bank: Washington, DC, USA, 2008; Volume 20, pp. 335-438.

17. Ben Romdhane, H.; Husseini, A.; Jabbour, S. Non-Communicable Diseases-II: Focus on Cardiovascular Diseases; Cambridge University Press: New York, NY, USA, 2012; pp. 164-177.

18. Atek, M.; Traissac, P.; El Ati, J.; Laid, Y.; Aounallah-Skhiri, H.; Eymard-Duvernay, S.; Mézimèche, N.; Bougatef, S.; Béji, C.; Boutekdjiret, L. Obesity and association with area of residence, gender and socio-economic factors in Algerian and Tunisian adults. PLoS ONE 2013, 8, e75640. [CrossRef]

19. Ben Halim, N.; Bouafif, N.B.A.; Romdhane, L.; Atig, R.K.B.; Chouchane, I.; Bouyacoub, Y.; Arfa, I.; Cherif, W.; Nouira, S.; Talmoudi, F. Consanguinity, endogamy, and genetic disorders in Tunisia. J. Community Genet. 2013, 4, 273-284. [CrossRef]

20. Romdhane, L.; Halim, N.B.; Rejeb, I.; Kefi, R.; Bouyacoub, Y.; Rekaya, M.B.; Messai, H.; Messaoud, O.; Riahi, Z.; Bonnet, C. Specific aspects of consanguinity: Some examples from the Tunisian population. J. Human Hered. 2014, 77, 167-174. [CrossRef] [PubMed]

21. Zakaria, D. Etude de L'endogamie D’origine Régionale, de la Distribution de la Consanguinité Apparente et du Comportement Intergénérationnel Dans le Choix Matrimonial en Tunisie. Intérêt des Noms de Famille et de L'isonymie Maritale. Ph.D. Thesis, Faculty of Science, University of Tunis, Tunis, Tunisia, 1999.

22. Ben Halim, N.; Hsouna, S.; Lasram, K.; Rejeb, I.; Walha, A.; Talmoudi, F.; Messai, H.; Sabrine Ben Brick, A.; Ouragini, H.; Cherif, W.; et al. Differential impact of consanguineous marriages on autosomal recessive diseases in Tunisia. Am. J. Hum. Biol. 2016, 28, 171-180. [CrossRef]

23. Romdhane, L.; Abdelhak, S.; Research Unit on Molecular Investigation of Genetic Orphan Diseases and Collaborators. Genetic diseases in the Tunisian population. Am. J. Med. Genet. A 2011, 155, 238-267. [CrossRef]

24. Kharrat, M.; Trabelsi, S.; Chaabouni, M.; Maazoul, F.; Kraoua, L.; Ben Jemaa, L.; Gandoura, N.; Barsaoui, S.; Morel, Y.; M’Rad, R.; et al. Only two mutations detected in 15 Tunisian patients with 11beta-hydroxylase deficiency: The p.Q356X and the novel p.G379V. Clin. Genet. 2010, 78, 398-401. [CrossRef] [PubMed]

25. Kallabi, F.; Hadj Salem, I.; Ben Salah, G.; Ben Turkia, H.; Ben Chehida, A.; Tebib, N.; Fakhfakh, F.; Kamoun, H. Molecular characterization of $\mathrm{X}$-linked adrenoleukodystrophy in a Tunisian family: Identification of a novel missense mutation in the ABCD1 gene. Neurodegener. Dis. 2013, 12, 207-211. [CrossRef]

26. Chakroun, A.; Ben Said, M.; Ennouri, A.; Achour, I.; Mnif, M.; Abid, M.; Fakhfakh, F.; Kamoun, H. Long-term clinical follow-up and molecular testing for diagnosis of the first Tunisian family with Alstrom syndrome. Eur. J. Med. Genet. 2016, 59, 444-451. [CrossRef]

27. Salah, G.B.; Salem, I.H.; Masmoudi, A.; Rhouma, B.B.; Turki, H.; Fakhfakh, F.; Ayadi, H.; Kamoun, H. Chromosomal instability associated with a novel BLM frameshift mutation (c. 1980-1982delAA) in two unrelated Tunisian families with Bloom syndrome. J. Eur. Acad. Dermatol. Venereol. 2014, 28, 1318-1323. [CrossRef] [PubMed]

28. Kek, H.E.H.; Wigren, E.; Jlizi, A.; Zahra, K.; Pellechia, D.; Vinciguerra, C.; Meddeb, B.; Elggaaied, A.B.A.; Gouider, E. First report of molecular diagnosis of Tunisian. hemophiliacs A: Identification of 8 novel. causative mutations. Diagn. Pathol. $2012,7,93$.

29. Cherif, W.; Turkia, B.; Rhouma, B.; Riahi, I.; Chemli, J.; Amaral, O.; Miranda, S.; Caillaud, C.; Kaabachi, N.; Tebib, N. Molecular diagnosis of Gaucher disease in Tunisia. Pathol. Biol. 2012, 61, 59-63. [CrossRef] [PubMed] 
30. Mazzucchelli, I.; Garofoli, F.; Decembrino, L.; Castiglia, D.; Tadini, G.; Bellingeri, A.; Borghesi, A.; Tzialla, C.; Manzoni, P.; Stronati, M. A novel LAMA3 mutation in a newborn with junctional epidermolysis bullosa herlitz type. Neonatology 2011, 99, 188-191. [CrossRef] [PubMed]

31. Khiari, H.M.; Lesca, G.; Malafosse, A.; Mrabet, A. A novel exon 3 mutation in a Tunisian patient with Lafora's disease. J. Neurol. Sci. 2011, 304, 136-137. [CrossRef] [PubMed]

32. Tinsa, F.; Ben Romdhane, M.; Boudabous, H.; Bel Hadj, I.; Brini, I.; Tebib, N.; Louati, H.; Bekri, S.; Boussetta, K. A Novel Mutation c.153 C > A in a Tunisian Girl with Wolman Disease and Unusual Presentation: Hemophagocytic Lymphohistiocytosis. J. Pediatr. Hematol. Oncol. 2018, 41, e193-e196. [CrossRef] [PubMed]

33. Jaafar, N.; Moleirinho, A.; Kerkeni, E.; Monastiri, K.; Seboui, H.; Amorim, A.; Prata, M.J.; Quental, S. Molecular characterization of maple syrup urine disease patients from Tunisia. Gene 2013, 517, 116-119. [CrossRef]

34. Valente, E.M.; Logan, C.V.; Mougou-Zerelli, S.; Lee, J.H.; Silhavy, J.L.; Brancati, F.; Iannicelli, M.; Travaglini, L.; Romani, S.; Illi, B.; et al. Mutations in TMEM216 perturb ciliogenesis and cause Joubert, Meckel and related syndromes. Nat. Genet. 2010, 42, 619-625. [CrossRef]

35. Mkaouar-Rebai, E.; Chamkha, I.; Mezghani, N.; Ben Ayed, I.; Fakhfakh, F. Screening of mitochondrial mutations in Tunisian patients with mitochondrial disorders: An overview study. Mitochondrial DNA 2013, 24, 163-178. [CrossRef]

36. Said, M.B.; Chouchène, E.; Salem, S.B.; Daoud, K.; Largueche, L.; Bouassida, W.; Benzina, Z.; Ayadi, H.; Söderkvist, P.; Matri, L.; et al. Posterior microphthalmia and nanophthalmia in Tunisia caused by a founder c.1059_1066insC mutation of the PRSS56 gene. Gene 2013, 528, 288-294. [CrossRef]

37. Rekik, S.; Sakka, S.; Romdhane, S.B.; Amer, Y.B.; Lehkim, L.; Farhat, N.; Mahfoudh, K.B.; Authier, F.J.; Dammak, M.; Mhiri, C. Novel splicing dysferlin mutation causing myopathy with intra-familial heterogeneity. Mol. Biol. Rep. 2020, 47, $5755-5761$. [CrossRef]

38. Chkioua, L.; Grissa, O.; Leban, N.; Gribaa, M.; Boudabous, H.; Turkia, H.B.; Ferchichi, S.; Tebib, N.; Laradi, S. The mutational spectrum of hunter syndrome reveals correlation between biochemical and clinical profiles in Tunisian patients. BMC Med. Genet. 2020, 21, 111. [CrossRef]

39. Ouesleti, S.; Brunel, V.; Ben Turkia, H.; Dranguet, H.; Miled, A.; Miladi, N.; Ben Dridi, M.F.; Lavoinne, A.; Saugier-Veber, P.; Bekri, S. Molecular characterization of MPS IIIA, MPS IIIB and MPS IIIC in Tunisian patients. Clin. Chim. Acta 2011, 412, 2326-2331. [CrossRef] [PubMed]

40. Chkioua, L.; Khedhiri, S.; Grissa, O.; Aloui, C.; Turkia, H.B.; Ferchichi, S.; Miled, A.; Froissart, R.; Acquaviva, C.; Laradi, S. Genetic basis of cystinosis in Tunisian patients: Identification of novel mutation in CTNS gene. Meta Gene 2015, 5, 144-149. [CrossRef] [PubMed]

41. Pohler, E.; Mamai, O.; Hirst, J.; Zamiri, M.; Horn, H.; Nomura, T.; Irvine, A.D.; Moran, B.; Wilson, N.J.; Smith, F.J.; et al. Haploinsufficiency for AAGAB causes clinically heterogeneous forms of punctate palmoplantar keratoderma. Nat. Genet. 2012, 44, 1272-1276. [CrossRef] [PubMed]

42. Romdhane, L.; Messaoud, O.; Bouyacoub, Y.; Kerkeni, E.; Naouali, C.; Cherif Ben Abdallah, L.; Tiar, A.; Charfeddine, C.; Monastiri, K.; Chabchoub, I.; et al. Comorbidity in the Tunisian population. Clin. Genet. 2016, 89, 312-319. [CrossRef]

43. Mrabet, H.; Borhani-Haghighi, A.; Koseoglu, E.; Mutlu, M.; Baydemir, R.; Nafissi, S.; Eschebbi, S.; Delibas, E.; Samangooie, S.; Yetkin, F.; et al. Association of amyotrophic lateral sclerosis and Behcet's disease: Is there a relationship? A multi-national case series. Clin. Rheumatol. 2012, 31, 733-738. [CrossRef]

44. Sayeb, M.R.Z.; Laroussi, N.; Bonnet, C.; Romdhane, L.; Mkaouar, R.; Zaouak, A.; Marrakchi, J.; Abdessalem, G.; Messaoud, O.; Bouchniba, O.; et al. A Tunisian family with a novel mutation in the gene CYP4F22 for lamellar ichthyosis and co-occurrence of hearing loss in a child due to mutation in the SLC26A4 gene. Int. Soc. Dermatol. 2019, 58, 1439-1443. [CrossRef]

45. Ghedira, N.; Lagarde, A.; Ben Ameur, K.; Elouej, S.; Sakka, R.; Kerkeni, E.; Chioukh, F.Z.; Olschwang, S.; Desvignes, J.P.; Abdelhak, S.; et al. Clinical profile of comorbidity of rare diseases in a Tunisian patient: A case report associating incontinentia pigmenti and Noonan syndrome. BMC Pediatr. 2018, 18, 286. [CrossRef]

46. Tabebi, M.; Charfi, N.; Kallabi, F.; Alila-Fersi, O.; Ben Mahmoud, A.; Tlili, A.; Keskes-Ammar, L.; Kamoun, H.; Abid, M.; Mnif, M.; et al. Whole mitochondrial genome screening of a family with maternally inherited diabetes and deafness (MIDD) associated with retinopathy: A putative haplotype associated to MIDD and a novel MT-CO2 m.8241T>G mutation. J. Diabetes Complicat. 2017, 31, 253-259. [CrossRef] [PubMed]

47. Baya, W.; Fredj, F.B.; Hassine, I.B.; Anoun, J.; Mzabi, A.; Karmani, M.; Rezgui, A.; Laatiri, M.A.; Kechrid, C.L. Systemic lupus erythematosus, antiphospholipid syndrome and Hashimoto thyroiditis occurring in a patient with Niemann-Pick disease: A second case. Pan Afr. Med. J. 2020, 36, 367. [CrossRef] [PubMed]

48. Louhichi, N.; Bahloul, E.; Marrakchi, S.; Othman, H.B.; Triki, C.; Aloulou, K.; Trabelsi, L.; Mahfouth, N.; Ayadi-Mnif, Z.; Keskes, L.; et al. Thyroid involvement in Chanarin-Dorfman syndrome in adults in the largest series of patients carrying the same founder mutation in ABHD5 gene. Orphanet J. Rare Dis. 2019, 14, 112. [CrossRef]

49. Charfeddine, C.; Dallali, H.; Abdessalem, G.; Ghedira, K.; Hamdi, Y.; Elouej, S.; Landoulsi, Z.; Delague, V.; Lagarde, A.; Levy, N.; et al. Identification of a CDH12 potential candidate genetic variant. for an autosomal dominant form of transgrediens and progrediens palmoplantar keratoderma in a Tunisian family. J. Hum. Genet. 2020, 65, 397-410. [CrossRef] [PubMed]

50. Ben Romdhan, S.; Sakka, S.; Farhat, N.; Triki, S.; Dammak, M.; Mhiri, C. A Novel SYNJ1 Mutation in a Tunisian Family with Juvenile Parkinson's Disease Associated with Epilepsy. J. Mol. Neurosci. 2018, 66, 273-278. [CrossRef] 
51. Blibech, R.; Gharbi, Y.; Mrad, A.; Zahra, H.; Mahjoub, T.; Belhaj, A.; Laatiri, Z.; Kastally, R.; Rosa, R. Incidence of glucose-6phosphate dehydrogenase (G6PD) deficiency in Tunisian populations. Nouv. Rev. Fr. d'Hématol. 1989, 31, $189-191$.

52. Fattoum, S. Evolution of hemoglobinopathy prevention in Africa: Results, problems and prospect. Mediterr. J. Hematol. Infect. Dis. 2009, 1, e2009005. [CrossRef]

53. Zilber Nelly, K.E.; Miriam, A. The Libyan Creutzfeldt-Jakob disease focus in Israel: An epidemiologic evaluation. Neurology 1991, 41, 1385. [CrossRef]

54. Boussetta, K.; Khalsi, F.; Bahri, Y.; Belhadj, I.; Tinsa, F.; Messaoud, T.B.; Hamouda, S. Cystic fibrosis in Tunisian children: A review of 32 children. Afr. Health Sci. 2018, 18, 664-670. [CrossRef]

55. Kharfi, M.; Khaled, A.; Mokhtar, I.; Kamoun, M. Atopic dermatitis in Tunisia: Epidemiological and clinical aspects. Ann. Dermatol. Venereol. 2001, 128, 623-625.

56. Brick, A.S.B.; Laroussi, N.; Mesrati, H.; Kefi, R.; Bchetnia, M.; Lasram, K.; Halim, N.B.; Romdhane, L.; Ouragini, H.; Marrakchi, S. Mutational founder effect in recessive dystrophic epidermolysis bullosa families from Southern Tunisia. Arch. Dermatol. Res. 2014, 306, 405-411. [CrossRef]

57. Ayed, S.; Ghorbel, M.; Nacef, L.; Daghfous, F.; Osman, N.B.; Reguig, R.; Lachhab, M.J.L.T.m. The exfoliation syndrome in Tunisia. Tunis. Med. 1990, 68, 19-22.

58. Ben Abdallah, M.; Zehani, S. Registre des Cancers Nord.-Tunisie 1995-1998; Institut Salah Azaiez: Tunis, Tunisia, 2004.

59. Frikha, M.; Mseddi, S.; Elloumi, M.; Bouaziz, M.; Khanfir, A.; Mnif, J.; Saad, A.; Souissi, T. Fanconi disease: Study of 43 cases in Southern Tunisia. Arch. Pediatr. Organe Off. Soc. Fr. Pediatr. 1998, 5, 1200-1205. [CrossRef]

60. Slimane, M.; Pousse, H.; Maatoug, F.; Hammami, M.; Farhat, M.B. Phenotypic expression of familial hypercholesterolaemia in central and southern Tunisia. Atherosclerosis 1993, 104, 153-158. [CrossRef]

61. Turkia, B.; Tebib, N.; Azzouz, H.; Abdelmoula, M.S.; Chehida, B.; Chemli, J.; Monastiri, K.; Chaabouni, M.; Sanhagi, H.; Zouari, B. Incidence of mucopolysaccharidoses in Tunisia. Tunis. Med. 2009, 87, 782-785.

62. Nasrallah, F.; Hadj-Taieb, S.; Chehida, A.B.; Jelassi, A.; Massoued, S.B.; Charfi, M.; Zidi, W.; Amri, F.; Helel, K.B.; Mejaoual, H. Nonketotic hyperglycinemia in tunisia. Report upon a series of 69 patients. Neuropediatrics 2020, 51, 349-353. [CrossRef] [PubMed]

63. Chehida, A.B.; Messaoud, S.B.; Abdelaziz, R.B.; Mansouri, H.; Boudabous, H.; Hakim, K.; Ali, N.B.; Ameur, Z.B.; Sassi, Y.; Kaabachi, N. A lower energetic, protein and uncooked cornstarch intake is associated with a more severe outcome in glycogen storage disease type III: An observational study of 50 patients. J. Pediatr. Endocrinol. Metab. 2018, 31, 979-986. [CrossRef] [PubMed]

64. Laradi, S.; Monastiri, K.; Ferchichi, S.; Nabli, N.; Rea, P.A.; Limam, H.B.; Mansour, R.B.; Bousoffara, R.; Yacoub, M.; Froissart, R. Les mucopolysaccharidoses (MPS) sont des maladies de surcharge lysosomiale. Ann. Biol. Clin. 2001, 59, $100-104$.

65. McNally, E.M.; Passos-Bueno, M.R.; Bönnemann, C.G.; Vainzof, M.; de Sa Moreira, E.; Lidov, H.; Othmane, K.B.; Denton, P.H.; Vance, J.M.; Zatz, M. Mild and severe muscular dystrophy caused by a single gamma-sarcoglycan mutation. Am. J. Hum. Genet. 1996, 59, 1040. [PubMed]

66. Moussa, S.A.-B.; Moussa, A.; Lovecchio, T.; Kourda, N.; Najjar, T.; Jilani, S.B.; El Gaaied, A.; Porchet, N.; Manai, M.; Buisine, M.-P. Identification and characterization of a novel MLH1 genomic rearrangement as the cause of HNPCC in a Tunisian family: Evidence for a homologous Alu-mediated recombination. Fam. Cancer 2009, 8, 119-126. [CrossRef]

67. Ben-Bassat, I.; Feinstein, A.; Ramot, B. Selective vitamin B12 malabsorption with proteinuria in Israel. Clinical and genetic aspects. Isr. J. Med. Sci. 1969, 5, 62-68.

68. Dridi, M.-F.B.; Turkia, H.B.; Azzouz, H.; Chehida, A.B.; Abdelaziz, R.B.; Tebib, N. Les maladies héréditaires du métabolisme en Tunisie: Défis, acquis, espoirs. Arch. Pediatr. Organe Off. Soc. Fr. Pediatr. 2015, 5, 3-4. [CrossRef]

69. Ben Rekaya, M.; Jerbi, M.; Messaoud, O.; Ben Brick, A.S.; Zghal, M.; Mbarek, C.; Chadli-Debbiche, A.; Jones, M.; Mokni, M.; Boussen, H.; et al. Further evidence of mutational heterogeneity of the XPC gene in Tunisian families: A spectrum of private and ethnic specific mutations. BioMed Res. Int. 2013, 2013, 316286. [CrossRef] [PubMed]

70. Ouali, F.; Siala, H.; Bibi, A.; Hadj Fredj, S.; Dakhlaoui, B.; Othmani, R.; Ouenniche, F.; Zouari, F.; Bouguerra, B.; Rezigua, H.; et al. Prenatal diagnosis of hemoglobinopathies in Tunisia: An 18 years of experience. Int. J. Lab. Hematol. 2016, 38, 223-232. [CrossRef] [PubMed]

71. Oueslati, S.; Hadj Fredj, S.; Belhaj, R.; Siala, H.; Bibi, A.; Messaoud, T. Preliminary study of haplotypes linked to the rare cystic fibrosis E1104X mutation. Acta Physiol. Hung. 2015, 102, 86-93. [CrossRef]

72. Abed, A.B.; Saad, H.; Mustpha, R.; Chiha, M.; Gamra, B. Early hearning screening by otoacoustic emissions and auditory brain stem response in Nabeul. Tunis. Med. 2013, 91, 643-647.

73. Guellouz, N.; Mansour, B.; Ouederni, M.; Jabnoun, S.; Kacem, S.; Ch, M.; Kastally, R.; Chahed, M.; Khrouf, N. Neonatal screening of G6PD deficiency in Tunisia. Arch. L'Inst. Pasteur Tunis 2010, 87, 69-76.

74. Hajer, S.; Neila, T.; Sondess, H.F.; Fekria, O.; Nabila, A.; Mahbouba, K.; Melika, D.; Faida, O.; Amina, B.; Raja, B. A lower-cost protocol for sickle cell disease neonatal screening in Tunisia. Ann. Saudi Med. 2012, 32, 49-52. [CrossRef] [PubMed]

75. Verma, I.C.; Puri, R.D. Global burden of genetic disease and the role of genetic screening. Semin. Fetal. Neonatal. Med. 2015, 20, 354-363. [CrossRef]

76. Zlotogora, J. Autosomal recessive diseases among the Israeli Arabs. Hum. Genet. 2019, 138, 1117-1122. [CrossRef] [PubMed] 
77. Messaoud, O.; Rekaya, M.B.; Ouragini, H.; Benfadhel, S.; Azaiez, H.; Kefi, R.; Gouider-Khouja, N.; Mokhtar, I.; Amouri, A.; Boubaker, M.S.; et al. Severe phenotypes in two Tunisian families with novel XPA mutations: Evidence for a correlation between mutation location and disease severity. Arch. Dermatol. Res. 2012, 304, 171-176. [CrossRef]

78. Zhang, F.; Guo, X.; Zhang, Y.; Wen, Y.; Wang, W.; Wang, S.; Yang, T.; Shen, H.; Chen, X.; Tian, Q. Genome-wide copy number variation study and gene expression analysis identify ABI3BP as a susceptibility gene for Kashin-Beck disease. J. Hum. Genet. 2014, 133, 793-799. [CrossRef]

79. Monies, D.; Abouelhoda, M.; AlSayed, M.; Alhassnan, Z.; Alotaibi, M.; Kayyali, H.; Al-Owain, M.; Shah, A.; Rahbeeni, Z.; Al-Muhaizea, M.A.J.H.g. The landscape of genetic diseases in Saudi Arabia based on the first 1000 diagnostic panels and exomes. Hum. Genet. 2017, 136, 921-939. [CrossRef] [PubMed]

80. Richmond, C.M.; Campbell, S.; Foo, H.W.; Lunke, S.; Stark, Z.; Moody, A.; Bannister, E.; Greenway, A.; Brown, N. Rapid Identification of Biallelic SPTB Mutation in a Neonate with Severe Congenital Hemolytic Anemia and Liver Failure. Mol. Syndr. 2020, 11, 50-55. [CrossRef]

81. Bouyacoub, Y.; Falfoul, Y.; Ouederni, M.; Sayeb, M.; Chedli, A.; Chargui, M.; Sassi, H.; Chakroun Chenguel, I.; Munier, F.L.; El Matri, L.; et al. Granular type I corneal dystrophy in a large consanguineous Tunisian family with homozygous p.R124S mutation in the TGFBI gene. Ophthalmic Genet. 2019, 40, 329-337. [CrossRef]

82. Sfar, S.; Bzéouich, A.A.; Kerkeni, E.; Bouaziz, S.; Najjar, M.F.; Chouchane, L.; Monastiri, K. A novel CASR mutation in a Tunisian FHH/NSHPT family associated with a mental retardation. Mol. Biol. Rep. 2012, 39, 2395-2400. [CrossRef]

83. John, J.N.; Sid, E.; Zhu, Q. Recurrent Neural Networks to Automatically Identify Rare Disease Epidemiologic Studies from PubMed. In Proceedings of the AMIA Annual Symposium Proceedings, San Diego, CA, USA, 30 October-3 November 2021; p. 325.

84. De Antonio, M.; Dogan, C.; Daidj, F.; Eymard, B.; Puymirat, J.; Mathieu, J.; Gagnon, C.; Katsahian, S.; Filnemus Myotonic Dystrophy Study Group; Hamroun, D.; et al. The DM-scope registry: A rare disease innovative framework bridging the gap between research and medical care. Orphanet J. Rare Dis. 2019, 14, 122. [CrossRef] [PubMed]

85. Zayed, H.; Ouhtit, A.; El Bekay, R. An Arab registry for type 1 diabetes: Global benefits for type 1 diabetes patients. Curr. Med. Res. Opin. 2016, 32, 1681-1684. [CrossRef]

86. Elmahmoudi, H.; Chalbi, A.; Ben-Lakhal, F.; Borji, W.; Zahra, K.; Zorgan, M.; Meddeb, B.; Gouider, E. Regional registry of bleeding disorders in Tunisia. J. Haemoph. 2012, 1, 3. [CrossRef]

87. Iourov, I.Y.; Vorsanova, S.G.; Yurov, Y.B.; Bertrand, T. VIII World Rett Syndrome Congress \& Symposium of rare diseases, Kazan, Russia. Mol. Cytogenet. 2018, 11, 61.

88. Ayed, F.F.-B.; Douira-Khomsi, W.; Rhayem, S.; Jelassi, M.; Zribi, H.; Chaabouni, M.; Khemiri, M.; Bellagha, I.; Barsaoui, S. Burkitt lymphoma in a child with Bloom syndrome. Arch. Pédiatr. 2016, 23, 382-384. [CrossRef]

89. Riahi, Z.; Chahed, H.; Jaafoura, H.; Zainine, R.; Messaoud, O.; Naili, M.; Nagara, M.; Hammami, H.; Laroussi, N.; Bouyacoub, Y. A novel frameshift mutation (c. 405delC) in the GJB2 gene associated with autosomal recessive hearing loss in two Tunisian families. Int. J. Pediatr. Otorhinolaryngol. 2013, 77, 1485-1488. [CrossRef] [PubMed]

90. Riahi, Z.; Hammami, H.; Ouragini, H.; Messai, H.; Zainine, R.; Bouyacoub, Y.; Romdhane, L.; Essaid, D.; Kefi, R.; Rhimi, M. Update of the spectrum of GJB2 gene mutations in Tunisian families with autosomal recessive nonsyndromic hearing loss. Gene 2013, 525, 1-4. [CrossRef] [PubMed]

91. Amaral, M. Novel personalized therapies for cystic fibrosis: Treating the basic defect in all patients. J. Intern. Med. 2015, 277, 155-166. [CrossRef] [PubMed]

92. Connett, G. Lumacaftor-ivacaftor in the treatment of cystic fibrosis: Design, development and place in therapy. Drug Des. Dev. Ther. 2019, 13, 2405-2412. [CrossRef]

93. Arora, K.; Yarlagadda, S.; Zhang, W.; Moon, C.; Bouquet, E.; Srinivasan, S.; Li, C.; Stokes, D.C.; Naren, A. Personalized medicine in cystic fibrosis: Genistein supplementation as a treatment option for patients with a rare S1045Y-CFTR mutation. Am. J. Physiol.-Lung Cell. Mol. Physiol. 2016, 311, L364-L374. [CrossRef] [PubMed]

94. Messaoud, T.; Bibi, A.; Elion, J.; Férec, C.; Fattoum, S. Molecular epidemiology of cystic fibrosis in Tunisia. Ann. Biol. Clin. 2005, $63,627-630$.

95. Zhang, X.; Hothi, J.S.; Zhang, Y.H.; Srinivasan, S.; Stokes, D.C.; Zhang, W. c. 3623G> A mutation encodes a CFTR protein with impaired channel function. Respir. Res. 2016, 17, 1-6. [CrossRef]

96. El Kerch, F.; Ratbi, I.; Sbiti, A.; Laarabi, F.Z.; Barkat, A.; Sefiani, A. Carrier frequency of the c.525delT mutation in the SGCG gene and estimated prevalence of limb girdle muscular dystrophy type $2 \mathrm{C}$ among the Moroccan population. Genet. Test. Mol. Biomark. 2014, 18, 253-256. [CrossRef]

97. Siala, O.; Kammoun Feki, F.; Louhichi, N.; Hadj Salem, I.; Gribaa, M.; Elghzel, H.; Saad, A.; Triki, C.; Fakhfakh, F. Molecular prenatal diagnosis of muscular dystrophies in Tunisia and postnatal follow-up role. Genet. Test. 2008, 12, 581-586. [CrossRef]

98. Herson, S.; Hentati, F.; Rigolet, A.; Behin, A.; Romero, N.B.; Leturcq, F.; Laforêt, P.; Maisonobe, T.; Amouri, R.; Haddad, H.J.B. A phase I trial of adeno-associated virus serotype $1-\gamma$-sarcoglycan gene therapy for limb girdle muscular dystrophy type $2 \mathrm{C}$. Brain 2012, 135, 483-492. [CrossRef] [PubMed]

99. Hamdi, Y.; Mighri, N.; Boujemaa, M.; Mejri, N.; Ben Nasr, S.; Ben Rekaya, M.; Messaoud, O.; Bouaziz, H.; Berrazega, Y.; Rachdi, H.; et al. Identification of Eleven Novel BRCA Mutations in Tunisia: Impact on the Clinical Management of BRCA Related Cancers. Front. Oncol. 2021, 11, 674965. [CrossRef] [PubMed] 
100. Brosco, J.P.; Grosse, S.D.; Ross, L.F. Universal state newborn screening programs can reduce health disparities. JAMA Pediatr. 2015, 169, 7-8. [CrossRef]

101. Heeju Sohna, S.T. Inequities in newborn screening: Race and the role of medicaid. SSM—Popul. Health 2019, 9, 100496. [CrossRef] [PubMed]

102. Hinton, C.F.; Mai, C.T.; Nabukera, S.K.; Botto, L.D.; Feuchtbaum, L.; Romitti, P.A.; Wang, Y.; Piper, K.N.; Olney, R.S. Developing a public health-tracking system for follow-up of newborn screening metabolic conditions: A four-state pilot project structure and initial findings. Genet. Med. 2014, 16, 484-490. [CrossRef]

103. Urv, T.K.; Parisi, M.A. Newborn Screening: Beyond the Spot. Rare Diseases Epidemiology: Update Overview; Springer: Berlin/Heidelberg, Germany, 2017; pp. 323-346. [CrossRef]

104. Romero, S.; Rink, B.; Biggio, J., Jr. Carrier screening in the age of genomic medicine: ACOG committee opinion, number 690. Obs. Gynecol. 2017, 129, e35-e40.

105. Ben-Shachar, R.; Svenson, A.; Goldberg, J.D.; Muzzey, D. A data-driven evaluation of the size and content of expanded carrier screening panels. Genet. Med. 2019, 21, 1931-1939. [CrossRef]

106. Krotoski, D.; Namaste, S.; Raouf, R.K.; El Nekhely, I.; Hindi-Alexander, M.; Engelson, G.; Hanson, J.W.; Howell, R.R.; Committee, M.N.S. Conference report: Second conference of the Middle East and North Africa newborn screening initiative: Partnerships for sustainable newborn screening infrastructure and research opportunities. Genet. Med. 2009, 11, 663-668. [CrossRef]

107. Teebi, A.S.; El-Shanti, H.I. Consanguinity: Implications for practice, research, and policy. J. Lancet 2006, 367, 970-971. [CrossRef]

108. Hsairi, M.; Mehdi, F.; Bellaaj, R.; Kassis, M.J.L.T. Health screening strategies in Maghreb countries: Situation Analysis and perspectives. Tunis. Med. 2018, 96, 688-695. [PubMed]

109. Mohamed, S.; Elsheikh, W.; Al-Aqeel, A.I.; Alhashem, A.M.; Alodaib, A.; Alahaideb, L.; Almashary, M.; Alharbi, F.; AlMalawi, H.; Ammari, A.; et al. Incidence of newborn screening disorders among 56632 infants in Central Saudi Arabia. A 6-year study. Saudi Med. J. 2020, 41, 703-708. [CrossRef] [PubMed]

110. Abdelmoktader, A.M. Risk factors for congenital hypothyroidism in Egypt: Results of a population case-control study (2003-2010). Ann. Saudi Med. 2013, 33, 273-276. [CrossRef] [PubMed]

111. Sadek, A.A.; Hassan, M.H.; Mohammed, N.A. Clinical and neuropsychological outcomes for children with phenylketonuria in Upper Egypt; a single-center study over 5 years. Neuropsychiatr. Dis. Treat. 2018, 14, 2551-2561. [CrossRef]

112. Sirdah, M.M.; Al-Kahlout, M.S.; Reading, N.S. National G6PD neonatal screening program in Gaza Strip of Palestine: Rationale, challenges and recommendations. Clin. Genet. 2016, 90, 191-198. [CrossRef]

113. Nouaili, E.B.H.; Chaouachi, S.; Bezine, A.; Hamadi, M.; Mbarek, C. Dépistage Systématique de la Surdité en Maternité par Oto-Emissions Acoustiques Provoquées (OTEAP): Etude Pilote. Tunis. Méd. 2010, 88, 482-485.

114. Raz, A.E.; Atar, M. Cousin marriage and premarital carrier matching in a Bedouin community in Israel: Attitudes, service development and educational intervention. BMJ. Sex. Reprod. Health 2004, 30, 49-51. [CrossRef]

115. Shiloh, S.; Reznik, H.; Bat-Miriam-Katznelson, M.; Goldman, B. Pre-marital genetic counselling to consanguineous couples: Attitudes, beliefs and decisions among counselled, noncounselled and unrelated couples in Israel. Soc. Sci. Med. 1995, 41, 1301-1310. [CrossRef]

116. Al Arrayed, S. Campaign to control genetic blood diseases in Bahrain. Public Health Genom. 2005, 8, 52-55. [CrossRef]

117. Al Arrayed, S.; Al Hajeri, A. Public awareness of sickle cell disease in Bahrain. Ann. Saudi Med. 2010, 30, 284-288. [CrossRef]

118. Zitouna, N.; Zekri, L.A.; Wardi, N.; Marrakchi, J.; Bouattour, Y.; Ben Hassine, H. Setting-up of a Science Shop project on the early detection of hearing impairment among preschool and school children. In Proceedings of the Inaugural Meeting of the Hearing Institute, Collège de France, Paris, France, 16-17 September 2019. 